\title{
Sinopsis del género Weinmannia (Cunoniaceae) en México y Centroamérica
}

\author{
por \\ J. Francisco Morales \\ Instituto Nacional de Biodiversidad (INBio), Apto. 22-3100, Santo Domingo de Heredia, Costa Rica
}

\begin{abstract}
Resumen
Morales, J.F. 2010. Sinopsis del género Weinmannia (Cunoniaceae) en México y Centroamérica. Anales Jard. Bot. Madrid 67(2): 137-155.

Las especies del género Weinmannia en México y Centroamérica se tratan en una sinopsis. Se reconocen diez especies $(W$. anisophylla, W. balbisiana, W. burserifolia, W. fagaroides, W. intermedia, W. karsteniana, W. pinnata, W. vulcanicola, W. wercklei), incluida la descripción de una nueva especie (W. horrida J.F. Morales). Se brinda una clave para las especies y para cada taxon descripciones, ilustraciones, datos de distribución, fenología y citación completa de especímenes examinados.
\end{abstract}

Palabras clave: México, Centro América, Cunoniaceae, Weinmannia.

Weinmannia L. (Cunoniaceae) es un género pantropical con alrededor de 150 especies, distribuidas principalmente en el neotrópico (Bernardi, 1961, 1963; Bradford, 1998; Harling, 1999; Stein, 2001). Taxonómicamente es un género difícil, sobre todo por la alta variación intraespecífica presente en la mayoría de las especies y por la falta de caracteres consistentes para separar los diferentes táxones. Bernardi (1961, 1963) publicó las últimas monografías de las especies del neotrópico, que son de utilidad reducida por la inconsistencia en las claves y falta de descripciones paralelas. De igual manera, algunos de los caracteres que fueron utilizados para delimitar algunos táxones, tales como el ancho (no la ausencia) de las alas del raquis son muy variables, incluso a veces dentro de un mismo individuo en el campo, por lo que su utilidad para separar especies es reducida. Finalmente, también se presentan problemas de tipo nomenclatural, ya que algunas de las secciones propuestas (p.ej., Simplicifoliae) fueron inválidamente publicadas por la no designación de un tipo. Con la excepción del tratamiento

\begin{abstract}
Morales, J.F. 2010. Sinopsis of the genus Weinmannia (Cunoniaceae) in Mexico and Central America. Anales Jard. Bot. Madrid 67(2): 137-155 (in Spanish).

A synopsis of Weinmannia of Mexico and Central America is presented. Ten species are recognized (W. anisophylla, W. balbisiana, W. burserifolia, W. fagaroides, W. intermedia, W. karsteniana, W. pinnata, W. vulcanicola, W. wercklei), including the description of a new species (W. horrida J.F. Morales). A key to the species, as well as descriptions, illustrations, distribution, phenological data, and specimens examined are given for each.
\end{abstract}

Keywords: Mexico, Central America, Cunoniaceae, Weinmannia.

de las Cunoniaceae para la Flora de Ecuador (Harling, 1999) y la Flora de la Guayana Venezolana (Bradford \& Berry, 1998), no se ha publicado ningún otro trabajo que clarifique la confusa situación imperante en las especies neotropicales de Weinmannia.

Como resultado de la preparación de las Cunoniaceae para el Proyecto de la Flora Mesoamericana, se presenta una sinopsis de las especies del género Weinmannia en México y Centroamérica. Para efectos prácticos, todas las especies se consideran como parte de la sección Weinmannia, dado que la sección Simplicifoliae, en el sentido propuesto por Bernardi (1963), no ha sido aproyada por análisis filogéneticos recientes (p.ej., Bradford, 1998, 2002).

\section{Notas sobre algunos caracteres mofológicos y uso de la clave}

Uno de los principales problemas en Weinmannia es la alta variabilidad morfológica que se presenta incluso en un mismo individuo, donde las hojas juveniles 

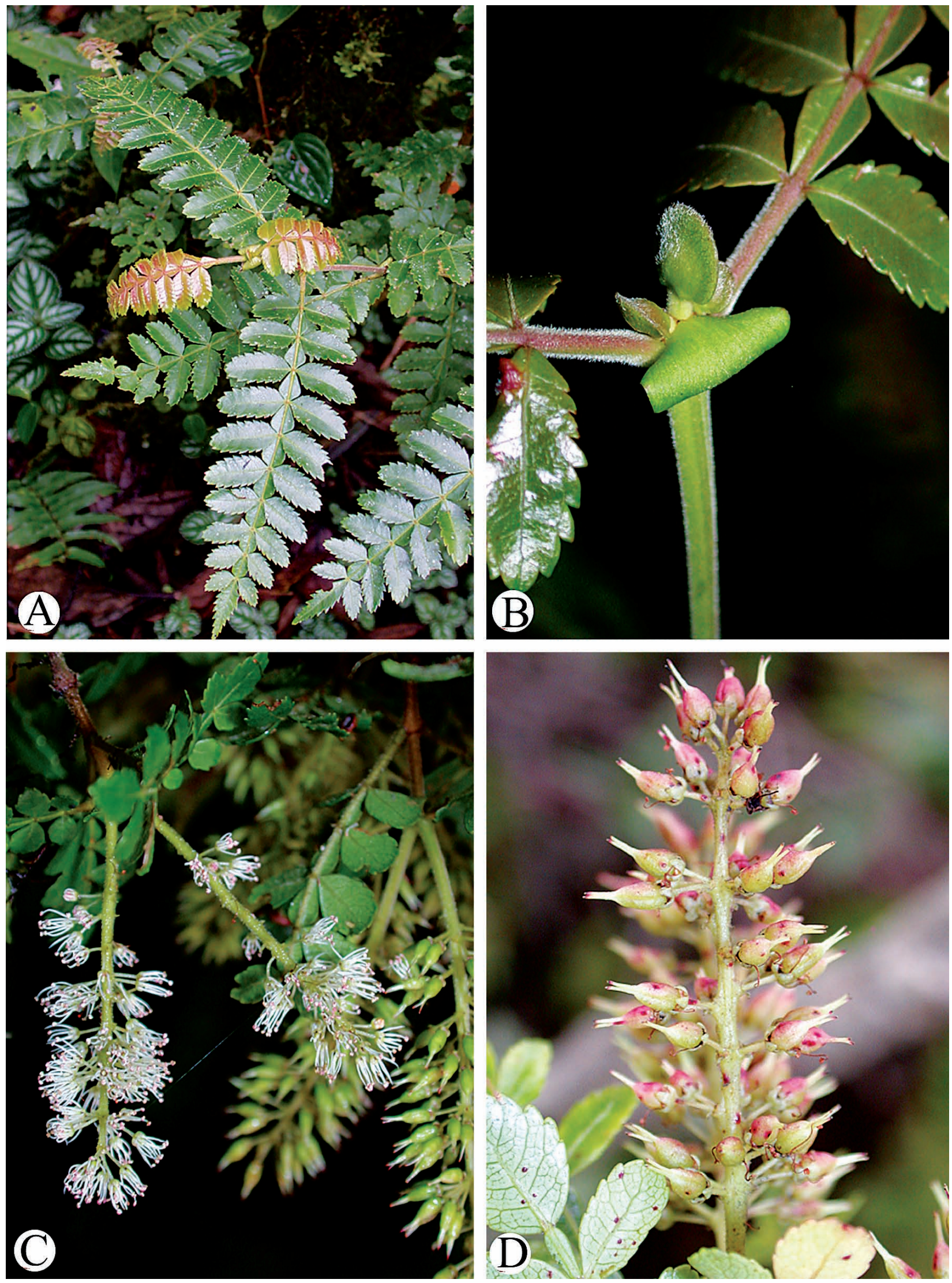

Fig. 1. Caracteres morfológicos en Weinmannia: A, hojas (W. intermedia); B, estípulas (W. pinnata); C, inflorescencias (W. pinnata); D, frutos (W. fagaroides) 
son en algunos casos muchísimo más grandes que las presentes en ramitas con flores o frutos. De igual manera, las inflorescencias y frutos son virtualmente idénticos en muchas especies, lo que dificulta la búsqueda de caracteres útiles para la separación a nivel intraespecífico. En muchos casos, la única forma de separar los diferentes táxones es basándose en el tamaño de los foliolos u hojas. Los principales caracteres morfológicos de Weinmannia son ilustrados en la Fig. 1.

La clave aquí propuesta ha sido diseñada para ser utilizada con especímenes fértiles: su uso en colecciones estériles puede llevar a identificaciones erróneas, dada la gran variabilidad en el tamaño de los foliolos en especímenes juveniles. Por lo tanto, aunque colecciones estériles casi no fueron examinadas ni tomadas en cuenta en la elaboración de las descripciones, en algunas de las discusiones se brindan pistas para el reconocimiento de material estéril. Sin embargo, es importante tener en cuenta que en todo el material de herbario examinado siempre se encuentran especímenes atípicos que no pueden ser ubicados satisfactoriamente a través de la clave y que representan estados intermedios entre dos táxones. Estos especímenes pueden ser el producto de la hibridación de diferentes especies, como ha sugerido Harling (1999), quien tuvo el mismo problema en el tratamiento de las especies del Ecuador.

En la clave y en descripciones, los términos "mayormente" o "usualmente" definen la predominancia de un carácter, pero no su exclusividad. De esta manera, el decir "hojas mayormente simples", se refiere a que en los especímenes predominan las hojas simples pero que, raramente, un nudo con hojas compuestas puede ser encontrado. Los extremos en el número de foliolos de las especies con hojas compuestas representan ámbitos que son raros u ocasionales en especímenes fértiles, pero nunca predominantes. De esta manera, por ejemplo, en Weinmannia intermedia el rango (7)13-21(25) indica que comúnmente la mayoría de las hojas tienen de 13 a 21 foliolos, pero que raramente unas pocas hojas pueden tener tantos como 25 o tan pocos como 7 .

En términos generales, las estípulas son virtualmente idénticas entre las diferentes especies presentes en México y Centroamérica. Es común que las estípulas totalmente maduras y desarrolladas no estén presentes en especímenes de herbario, sino solamente estípulas inmaduras. Por esta razón, las medidas brindadas en las descripciones están basadas en las estípulas de los especímenes herborizados, por lo que es probable que las medidas de aquellas presentes en especímenes vivos sean ligeramente mayores que los rangos aquí presentados.
Los pétalos son rápidamente deciduos después de la antesis; por tal razón, es usual que éstos no estén preservados en especímenes de herbario. La medida citada en las descripciones está basada en pétalos de botones florales aún sin abrir, por lo que sus dimensiones pueden diferir ligeramente de la presente en especímenes frescos. La medida de los frutos no incluye el estilo apical.

Una descripción detallada de los caracteres genéricos, así como de sinónimos nomenclaturales, puede ser encontrada en Harling (1999). En la presente sinopsis, un total de 10 especies son reconocidas en México y Centroamérica. Weinmmania tomentosa L.f. fue reportada dudosamente para México por Bernardi (1961), pero el testigo citado carece de una citación de localidad que afirme su procedencia; de todos modos, esa especie está restringida a Sudamérica y hasta el momento no se ha recolectado en ningún sitio de Centroamérica o México. De igual manera, en el mismo trabajo se reportó la presencia de W. trianaea Wedd. var. sulcata (Engl.) Cuatrec. en Costa Rica, pero Morales (2005) demostró que ese taxon está restringido al $\mathrm{N}$ de Sudamérica y que las poblaciones de Costa Rica representaban una especie sin describir (W. vulcanicola J. F. Morales).

\section{Clave PARA LAS ESPECIES Del GÉNeRo WeINMANNIA en MÉXICO Y CENTROAMÉRICA}

1. Hojas mayormente simples, a veces unas pocas hojas irregularmente 2- o 3-auriculadas, 2-folioladas o irregularmente 3folioladas en la misma rama (W. anisophylla) ................... 2

1. Hojas mayormente compuestas, algunas veces unas pocas hojas simples presentes en los ramos floríferos (W. anisophylla)

2. Ramitas con hojas simples y unas pocas compuestas presentes en la misma rama; hojas simples 2- o 3-auriculadas algunas veces también presentes; ovario glabro

1. W. anisophylla

2. Ramitas estrictamente con hojas simples; hojas simples lobuladas ausentes; ovario variadamente pubescente .............. 3

3. Láminas foliares $0,9-2,2 \times 0,7-1,6 \mathrm{~cm}$, la lámina suborbicular 7. W. karsteniana

3. Láminas foliares $2,5-12 \times 1-6,2 \mathrm{~cm}$, la lámina elíptica a angostamente elíptica mente en algunas pocas hojas inferiores a $2,2 \mathrm{~cm}$ de ancho, elípticas; peciolos hasta $4 \mathrm{~mm}$ de largo; cápsulas 3-4,5 mm de largo

2. W. balbisiana

4. Láminas foliares usualmente $1-2(2,5) \mathrm{cm}$ de ancho, raramente en algunas pocas hojas de más de $2 \mathrm{~cm}$ de ancho, angostamente elípticas; peciolos hasta $2 \mathrm{~mm}$ de largo; cápsulas 2$2,5(3) \mathrm{mm}$ de largo

10. W. wercklei

5. Hojas mayormente con (1)3-5(7) foliolos, de longitudes bastante diferentes entre sí, hojas simples usualmente presentes en los ramos floríferos ......................... 1. W. anisophylla

5. Hojas mayormente con más de 5 foliolos, de longitudes relativamente uniformes (excepto el foliolo terminal), hojas simples nunca presentes 
6. Foliolos usualmente con la lámina $0,4-0,8(1) \mathrm{cm}$ de largo, usualmente glabra, el margen entero, subentero o levemente crenado en el tercio apical ......... 4. W. fagaroides

6. Foliolos usualmente con la lámina $(0,5-) 0,8-4,5(-5,5) \mathrm{cm}$ de largo, glabra a pubescente, el margen evidentemente crenado, crenado-serrado o serrado en casi toda su longitud .... 7

7. Hojas más largas de las ramas floríferas en promedio usualmente con 13-25(29) foliolos

7. Hojas más largas de las ramas floríferas en promedio usualmente con 5-11(13) foliolos

8. Raquis e inflorescencias densamente hirsutas o hirsútulas, el indumento amarillo, los tricomas usualmente $1.5-2 \mathrm{~mm}$ de largo, erectos o suberectos; foliolos con la superficie abaxial hirsuta, hirsútula o más raramente glabrescente y con la pubescencia restringida principalmente al nervio central, el indumento erecto

5. W. horrida

8. Raquis de las hojas e inflorescencias, glabros, glabrescentes o variadamente puberulentos, pero el indumento no hirsuto o hirsútulo, si amarillento, entonces adpreso, los tricomas menos de $1 \mathrm{~mm}$ de largo, adpresos; superficie abaxial de la lámina de las hojas glabra, glabrescente o puberulenta (W. intermedia), pero entonces el indumento no hirsuto, hirsútulo o erecto

9. Indumento de los tallos ferrugíneo o café ferrugíneo; foliolos con los márgenes usualmente cóncavos y revolutos

9. W. vulcanicola

9. Indumento de los tallos algo amarillento o del mismo color de las ramitas; foliolos planos, los márgenes no cóncavos ni revolutos 10

10. Hojas mayormente con 5-11 foliolos, a veces unas pocas con 15 o 17 foliolos; cápsulas 3-3,5 mm de largo

8. W. pinnata

10. Hojas mayormente con 13-21 foliolos, a veces unas pocas con 11 foliolos; cápsulas 2-2,5 mm de largo

6. W. intermedia

11. Raquis de las hojas e inflorescencias densamente hirsutas o hirsútulas, el indumento amarillo, los tricomas usualmente 1,5-2 mm de largo, erectos o suberectos; foliolos con la superficie abaxial hirsuta, hirsútula o más raramente glabrescente y con la pubescencia restringida principalmente al nervio central, el indumento erecto

5. W. horrida

11. Raquis de las hojas e inflorescencias, glabros, glabrescentes o variadamente puberulentos, el indumento no hirsuto o hirsútulo, si amarillento, entonces adpreso, los tricomas menos de $1 \mathrm{~mm}$ de largo, adpresos; foliolos con la superficie abaxial glabra o glabrescente (raramente con el nervio central pubescente)

12. Foliolos con la lámina mayormente $(1,8) 2-4,5(5,5) \times$ $(0,9) 1-1,6 \mathrm{~cm}$; peciolos (9)12-17(23) mm de largo

3. W. burserifolia

12. Foliolos con la lámina mayormente $0,8-1,8(2,1) \times 0,4-1,1$ $\mathrm{cm}$; peciolos 6-11 $\mathrm{mm}$ de largo

8. W. pinnata

1. Weinmannia anisophylla Standl. \& L.O. Williams, Ceiba 3(1): 45. 1952

Tipo: Costa Rica. Alajuela: Palmira, Alfaro Ruiz, 2300 m, 18-IV-1940 (fl), Smith 2622 (holótipo, F [foto F neg 59235, fotocopia, INB]; isótipos, CR, EAP [fragmento], NY, US). Fig. 2.

Árboles ó arbustos de 1-10 m de altura, los tallos muy esparcidamente puberulentos a glabrescentes, indumento del mismo color del tallo, nunca ferrugí- neo o amarillento. Hojas simples a compuestas en la misma rama, las compuestas con 3-5(7) foliolos; lámina de (8)11-62(81) × (7)10-25(37) mm, elíptica, ovado-elíptica a ovada, membranácea, usualmente glabra o glabrescente y con unos cuantos tricomas esparcidos a lo largo del nervio central en la superficie abaxial, el ápice agudo a obtuso, la base obtusa a anchamente cuneada, los márgenes serrados o crenado-serrados, no revolutos; entrenudos del raquis en hojas compuestas alados, las alas de 3-8 $\mathrm{mm}$ de ancho, glabros o inconspicuamente puberulentos a lo largo del nervio central abaxialmente, el indumento del mismo color del tallo, nunca ferrugíneo o amarillento; peciolos 9-18 mm de largo, raramente 1-2 m en hojas simples, usualmente alados en la mitad distal, glabrescentes o glabros; estípulas inmaduras 6-9 × 5-8 mm, suborbiculares o anchamente ovadas. Inflorescencias $5,5-12 \mathrm{~cm}$ de largo, el raquis y los pedicelos glabrescentes, el indumento no ferrugíneo ni amarillento, inconspicuo; flores 2-2,5 mm de largo, pedicelos 1,22,7 mm de largo, lóbulos del cáliz 0,7-0,9 mm de largo, agudos, glabros, pétalos 1-1,3 mm de largo, ovario

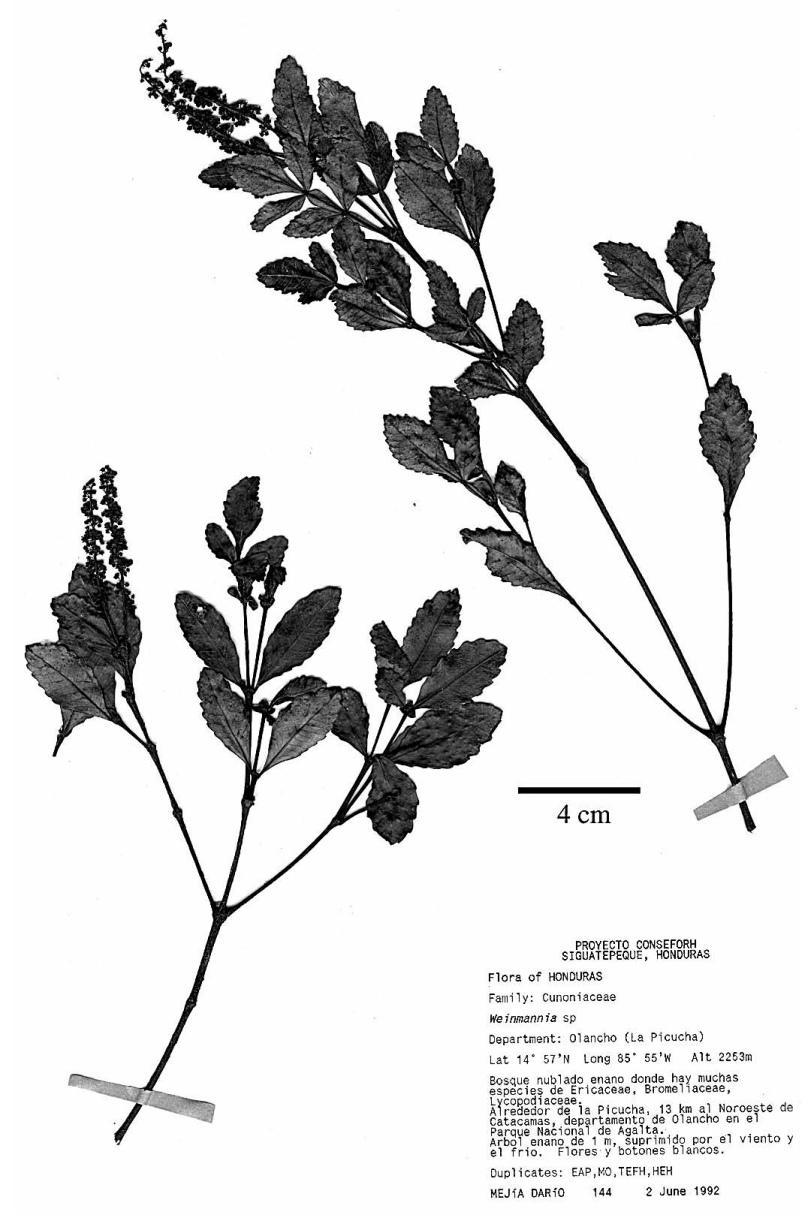

Fig. 2. Weinmannia anisophylla (Darío 144, INB). 
0,6-0,9 mm de largo, glabro. Cápsulas 2-3 mm de largo, glabras.

Distribución, hábitat y ecología. Conocida por pocas colecciones en Guatemala, Honduras, Costa Rica, y disyuntamente en Venezuela y Perú, donde crece en bosques nubosos, bosques en zonas ventosas y áreas de vegetación alterada entre los 2000 y $2400 \mathrm{~m}$. Floración y fructificación ocurre nentre abril y mayo.

Weinmannia anisopbylla aparentemente no está cercanamente relacionada con ninguna otra especie en México o Centroamérica y se puede reconocer con facilidad por la presencia usual de hojas simples y compuestas [de 3-5(7) foliolos] en la misma rama, con los foliolos de longitudes bastante diferentes, algunas veces con el foliolo terminal variadamente fusionado con alguno de los foliolos adyacentes. Por otro lado, es algo similar a $W$. auriculifera Hieron., conocida de Ecuador, pero esta última especie se distingue por sus hojas mucho más coriáceas y con la venación secundaria prominente y conspicua en el envés. De manera ocasional y sobretodo en especímenes estériles, $W$. wercklei Standl. tiene algún nudo con una hoja compuesta, pero esta última especie se puede diferenciar por la pubescencia más densa de sus ramitas tiernas. Estudios monográficos posteriores podrían demostrar que $W$. anisopbylla puede corresponder a un extremo anormal de la variable $W$. balbisiana, pero por ahora prefiero considerarlas como táxones distintos.

\section{Especímenes examinados}

GUATEMALA. Zacapa: Volcán Gemelos, 26-I-1942, Steyermark $43276(\mathrm{~F})$.

HONDURAS. Morazán: Montaña de Tigra, SW de San Juancito, 23-IV-1964, Molina 13743 (EAP, F); San Juancito, 25-III-1951, Williams 17526 (F), 10 -IV-1954, Williams \& Williams 18910 (EAP, TEFH). Olancho: Parque Nacional de Agalta, 31-V-1992, Dario 127 (EAP, INB, MO, TEFH); La Picucha, NE de Catacamas, 2-VI-1992, Dario 144 (EAP, INB, MO, TEFH); Sierra de Agalta, al E de Gualaco, 6-16-III-1997, Nelson E al. 19138 (TEFH).

COSTA RICA. San José: San Gerardo de Dota, Reserva Forestal Los Santos,-VI-1996, van Omme 234 (INB).

2. Weinmannia balbisiana Kunth, Nov. Gen. Sp. (quarto ed.) 6: 51, t. 520. 1823

Tipo: Perú. "Crescit in sylvis prope Loxa Peruvianorum”, (fl), Humboldt E Bonpland s.n. (holótipo, PHB). Fig. 3.

Weinmannia laurina Kunth, Nov. Gen. Sp. (quarto ed.) 6:51-52. 1823, syn. nov. Weinmannia balbisiana var. laurina (Kunth) Cuatrec., Lloydia 11: 201. 1948

Tipo: Ecuador. Sin datos (fl), Humboldt \& Bonpland s.n. (holótipo, P-HB, [fotografía F neg. 34640]).
Weinmannia tuerckbeimii Engl., Nat. Pflanzenfam. (ed. 2) 18a: 252. 1930, syn. nov.

Tipo: Guatemala. Alta Verapaz: entre Cobán y Samac, 1500 m, V-1886 (fl), von Tuerckbeim 906 (holótipo, $\mathrm{B}$; isótipo, $\mathrm{US}$ ).

Weinmannia pseudolaurina Woodson, Ann. Missouri Bot. Gard. 28(4): 429. 1941. Weinmannia laurina var. pseudolaurina (Woodson) Bernardi, Candollea 18:318. 1963

Tipo: Panamá. Panamá: Cerro Campana, sendero de Campana a Chica, 10-VIII-1941 (fr), Allen 2656 (holótipo, MO; isótipos, EAP, US).

Árboles o arbustos 2-7 m, tallos esparcidamente puberulentos, glabrescentes con la edad, el indumento del mismo color del tallo, no ferrugíneo ni amarillento. Hojas simples; lámina 30-120 × (15)22-62 mm, elíptica, membranácea, glabra a glabrescente, el ápice acuminado, agudo, obtuso-apiculado a obtuso o redondeado, la base obtusa o anchamente aguda, los márgenes crenado-serrados o serrados, a veces muy levemente y pareciendo subenteros, no revolutas; pe-

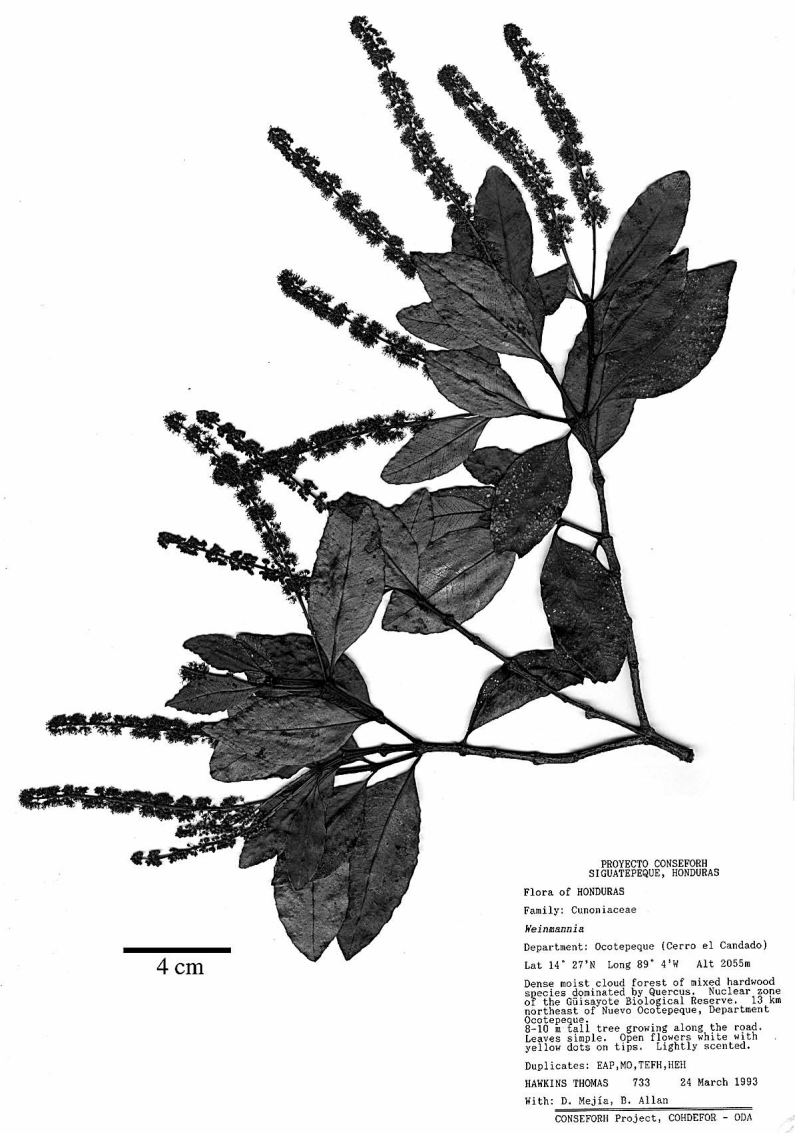

Fig. 3. Weinmannia balbisiana (Thomas et al. 733, INB). 
ciolos hasta $4 \mathrm{~mm}$ de largo, usualmente no alados, esparcidamente puberulentos a glabrescentes; estípulas 6,5-8 $\times$ 5-6 mm, suborbiculares. Inflorescencias (3) 4,5-13(16) $\mathrm{cm}$ de largo, el raquis y los pedicelos moderada a esparcidamente puberulentos, el indumento no ferrugíneo ni amarillento, inconspicuo; flores 2-2,5 mm de largo, pedicelos 2-4 mm de largo, lóbulos del cáliz 0,7-1 mm de largo, el ápice agudo, inconspicuamente puberulentos a glabros o glabrescentes, pétalos 1,3-1,9 mm de largo, ovario ca. $1 \mathrm{~mm}$ de largo, densamente puberulento. Cápsulas 3-4,5 mm de largo, diminuta y densamente puberulentas cuando jóvenes, glabrescentes con la edad.

Distribución, hábitat y ecología. México, Guatemala, Honduras, El Salvador, Costa Rica a Venezuela y Bolivia, donde crece en bosques muy húmedos, robledales, márgenes de ríos y áreas de vegetación secundaria relacionada entre los 1000-2300(2800) m (en Centroamérica). Especímenes con flores han sido recolectados entre febrero y junio y en septiembre. Material con frutos se ha colectado entre abril y octubre.

Weinmannia balbisiana puede ser confundida con W. wercklei Standl., ya que ambas tienen hojas simples y rangos de distribución altitudinal que se traslapan entre los 1000-2000 m; sin embargo, W. balbisiana se puede reconocer por sus hojas usualmente mucho más anchas $[(1,5-) 2,2-6,2 \mathrm{~cm}$ vs. $1-2(2,5) \mathrm{cm}]$ y frutos ligeramente más largos [3-4,5 mm vs. 2-2,5(3) $\mathrm{mm}]$. Los rangos extremos aquí expresados para ambas especies en relación al ancho de la lámina foliar son en ambos casos poco comunes, es decir, las hojas en especímenes con flores de W. wercklei raramente exceden los $2 \mathrm{~cm}$ de ancho, mientras que en $W$. balbisiana es común que midan más de $2.2 \mathrm{~cm}$ de ancho. Es común que $W$. balbisiana tenga hojas más reducidas cuando se encuentre creciendo sobre los $2500 \mathrm{~m}$, pero nunca tan angostas como las de W. wercklei.

En forma general, las plantas de W. balbisiana de zonas sobre los $2000 \mathrm{~m}$ tienen hojas mucho más pequeñas con relación a aquellas de áreas entre los 1000$1600 \mathrm{~m}$. Esta especie es un taxon altamente variable en la morfología foliar, lo que ha provocado que un sinfín de variedades o formas extremas hayan sido descritas a través de los años, principalmente en Colombia (p.ej., Cuatrecasas, 1948). Tomando en cuenta la variación presente en el NW de Sudamérica, lo cual también se presenta en las colecciones de Costa Rica y Panamá, no existe razón alguna que justifique el mantener la validez de Weinmannia tuerckheimii Engl. como un taxon aparte, por lo cual es reducido a la sinonimia, afirmando lo propuesto anteriormente por Bernardi (1963).

\section{Especímenes examinados}

MÉXICO. Oaxaca: vía Ixtlán a Valle Nacional, $\mathrm{N}$ del Cerro Humo Chico, 26-IX-1982, Cedillo \& Torres 1867 (MEXU, MO).

GUATEMALA. Alta Verapaz: E de Tactic, 14-IV-1941, Standley 92313 (F, G). Baja Verapaz: Purulhá, Biotopo del Quetzal, 12IX-1996, García \& al. 5043 (BIGU, INB); Niño Perdido, La Cumbre de San José Espinero, 10-V-1977, Lundell E Contreras 20907 (MO), 20-V-1977, Lundell \& Contreras 20930 (MO). El Progreso: Montaña Piamonte, 7-II-1942, Steyermark 43686 (EAP, F). Huehuetenango: Cerro Cananá, entre Nucapuxlac y Cananá, Sierra de los Cuchumatanes, 18-VII-1942, Steyermark 49002 (F). Zacapa: Monte Virgen, 12-I-1942, Steyermark 42617 (F).

HONDURAS. Comayagua: Campo Don Thomas, SW de Gracias, 11-V-1992, D'Arcy 17892 (EAP, INB, MO, TEFH); Cordillera de Montecillos, Montaña de San Juanillo, 10-V-1991, Davidse 34273 (EAP, INB, MO); cerro Azul de Meambar, 11-VIII-1974 (st), Hazlett 1900 (EAP); cerro Comayagua, 6-III-1975, Hazlett 2506 (MO); Cerro Azul, E del Lago Yojoa, 12-III-1993, Hawkins E al. 592 (EAP, MO, TEFH). Cortés: entre Cerro Cantiles y Cerro Jilinco, 19-III-1993, Mejía 340 (EAP, MO, TEFH). Lempira: río Naranjo, Parque Nacional Celaque, 9-VII-1991, House 1034 (EAP, MEXU, MO, TEFH). Morazán: San Juancito, 19-VI-1948, Glassman 1671 (EAP, F); Floresta de Nubes, Rancho Quemado,VI-1948, Molina 1007 (EAP, F, MO); Montaña La Tigra, SW de San Juancito, 14-VII-1961, Molina 10107 (EAP, F), 31-V-1962, Molina 10669 (EAP, F); SW de San Juancito, 11-VI-1963, Molina 12740 (EAP, F); Montaña La Tigra, 13-VII-1964, Molina 14500 (EAP, F); San Juancito, 21-V-1947, Williams E Molina 12739 (EAP, F, MEXU, MO), 20-II-1948, Williams \& Molina 13736 (EAP, F). Ocotepeque: Cerro El Candado, reserva biológica Güisayote, 24-III-1993, Hawkins \& al. 733 (EAP, INB, MO, TEFH); reserva biológica Güisayote, 24-III-1993, Thomas 733 (MO). Olancho: Parque Nacional Asalta, NE de Catacamas, 31-V-1992, Darío 128 (EAP, MO, TEFH).

EL SALVADOR. Chalatenango: Los Esesmiles, 4-IV-1942, Tucker 1201 (EAP, F, G, UC). Santa Ana: Cerro Montecristo, 19III-1959, Allen \& Armour 7286 (EAP, F, MO); cerro Montecristo, 4-6-II-1954 (st), Heed s.n. (EAP); montaña Montecristo, 18-III1977 (fl), Martínez s.n. (EAP, MHES); Montecristo, 23-V-1963, Molina E Molina 12665 (EAP, F); Montecristo, 4-VI-1976, Reyna s.n. (CR).

COSTA RICA. Cartago: Alto La Gloria, 29-III-1980, GómezLaurito 5274 (CR); Guarco, fecha perdida, González s.n. (F); río Grande de Orosí, al S de Tapantí, 5-XI-1967, Lent 1461 (CR, F); Parque Nacional Tapantí, Tausito, 6 -IV-1994, Morales É Lépiz 2636 (INB, MO, QCNE); Tapantí, 7-XII-1951, Reark 207 (CR); Paraíso, Orosí, Tausito, 11-II-1999, Rodríguez E Hurtado 4410 (CR, INB, MO); turbera La Chonta, 5-VI-1993, Vargas \& al. 1390 (CR); Parque Nacional Tapantí, Puente Dos Amigos, 22-X-1985, Zamora 1119 (CR, MO). Limón: Parque Internacional La Amistad, 21-VII-2000, Alfaro 3278 (INB); Cordillera de Talamanca, entre el río Terbi y el río Siní, 11-IX-1984, Davidse E al. 28956 (INB, MO), Davidse E al. 25128 (CR, MO). Puntarenas: Fila Anguciana, 17-VIII-2001, Alfaro 3700 (INB, MO); Parrita, Fila Chonta, 20-II1999, Morales E al. 6980 (INB). San José: entre La Gloria y La Cangreja, 21-II-1988, Burger \& al. 12399 (CR, F, MO); S de San Isidro del Guarco, 20-II-1988, Jiménez E al. 556 (CR, F, MO); Tarrazú, Cerro El Pito, 28-II-1995, Morales 3626 (INB, MO); Tarrazú, Cerro Hormiguero, 5-IX-1996, Morales \& al. 5761 (CR, INB, MO).

PANAMÁ. Bocas del Toro: Fortuna, 18-VI-1984, Churchill 5518 (MO); Cordillera de Talamanca, NW del Cerro Echandi, 23-III-1984, Davidse E al. 25193 (INB, MO); entre Boquete y Cerro Pate Macho, 7-II-1986, McPherson \& Merello 8332 (MO); Fortuna, 11-II-1986, McPherson 8403 (MO). Chiriquí: entre Chiriquí y 
Bocal, 9-II-1979, Hammel 6082 (MO); NE de Boquete, cerca de Palo Alto, 23-V-1979, Hammel 7379 (MO); Palo Alto, NE de Boquete, 27-V-1979, Hammel 7554 (MO); Cerro Colorado, N de San Félix, 3-V-1975, Mori \& Kallunki 5820 (MO), 18-VIII-1975, Mori E Dressler 7836 (MO); Bugaba, Cerro Punta, 26-I-1985, van der Werff E Herrera 6445 (MO). Darién: Cerro Pire, 14-XII-1962, Duke 6568 (MO); Cerro Mali, 16-I-1975, Gentry \& Mori 13623 (MO); pico W del Cerro Tacarcuna, 28-I-1975, Gentry \& Mori 13987 (MO); cima del Cerro Tacarcuna, 22-VII-1976, Gentry \& al. 16908 (MO); Alturas de Nique, 31-XII-1980, Hartman 12468 (MO); Cerro Pirre, 7-II-1991, Herrera E al. 888 (MO). Panamá: Cerro Campana, 31-XII-1985, McPherson 7899 (MO); Cerro Campana, 8-VIII-1975, Mori \& Bolten 7686 (MO).

3. Weinmannia burserifolia Standl., J. Wash. Acad. Sci. 17(12): 310. 1927

Tipo: Costa Rica. Heredia: Yerba Buena, NE de San Isidro, 2000 m, 28-II-1926 (fl), Standley E Valerio 49848 (holótipo, US). Fig. 4.

Weinmannia liebmannii Engl., Nat. Pflanzenfam. (ed. 2): 18a: 254. 1930

Tipo: México. Oaxaca: Pelado, VIII-1842 (fl, fr), Liebmann 1778 (holótipo, B; isótipo, C [2 pliegos, fotografías, INB].

Árboles o arbustos de 2,5-15 m de altura, los tallos esparcidamente puberulentos a glabros a glabrescentes (raramente moderada a densamente pubescentes), el indumento del mismo color del tallo. Hojas compuestas, con 5-11(13) foliolos; lámina (18)20-45(55) × (9)10-16 mm, elíptica a ovado-elíptica, membranácea a subcoriácea, glabra, raramente glabrescente o con el nervio central pubescente en la superficie abaxial, el ápice agudo, acuminado a obtuso o redondeado, la base obtusa o cuneada, los márgenes crenados, crenado-serrados o serrados, no revolutos; entrenudos del raquis alados, las alas $2-8 \mathrm{~mm}$ de ancho, el raquis glabrescente, usualmente con solo grupos de pelos en los nudos, el indumento incoloro; peciolos (8)12-17(23) $\mathrm{mm}$ de largo, glabros o glabrescentes, alados distalmente o no alados; estípulas 6-9 × 7-9 mm, suborbiculares. Inflorescencias 4-9,5(16) cm de largo, el raquis glabrescente o puberulento, los pedicelos esparcidamente adpreso-pubescentes, el indumento no amarillo ni ferrugíneo, inconspicuo; flores $2-2,5 \mathrm{~mm}$ de largo, pedicelos 2-3,5(5,5) $\mathrm{mm}$ de largo, lóbulos del cáliz 0,6-0,9 mm de largo, usualmente glabrescentes, el ápice agudo, pétalos ca. $2 \mathrm{~mm}$ de largo, ovario 1-1,4 mm de largo, glabro. Cápsulas 3-3,5 mm de largo, glabras.

Distribución, bábitat y ecología. Disyunta entre el $\mathrm{S}$ de México, Nicaragua, Costa Rica y el W de Panamá, donde crece en bosques muy húmedos, bosques enanos y nubosos, entre los 800-2800(3000) m de elevación. Especímenes con flores y frutos han sido recolectados en forma intermitente entre febrero y noviembre.

Weinmannia burserifolia se ha confundido y sinonimizado alternativamente en varios tratamientos con la común W. pinnata L (Bernardi, 1961; Woodson \& Schery, 1950). Sin embargo, puede ser separada con facilidad por los caracteres dados en la clave. En todo caso, algunas veces se encuentran en especímenes de herbario estados intermedios algo difíciles de separar, sobretodo en muestras vegetativas estériles. Weinmannia burserifolia está algo relacionada con W. lentiscifolia C. Presl, restringida al NW de Sudamérica, pero este último taxon tiene usualmente mucho más foliolos. El concepto de W. glabra L. f. usado en el tratamiento de la Flora de Nicaragua por Stein (2001) es el mismo utilizado aquí; sin embargo, W. glabra es un nombre ilegítimo, basado en el mismo tipo de W.pinnata y, por lo tanto, no debe usarse. Algunas colecciones de Oaxaca, México, tienen tallos notablemente pubescentes y las hojas con la mitad proximal del nervio central pubescente. Esta variedad fue descrita

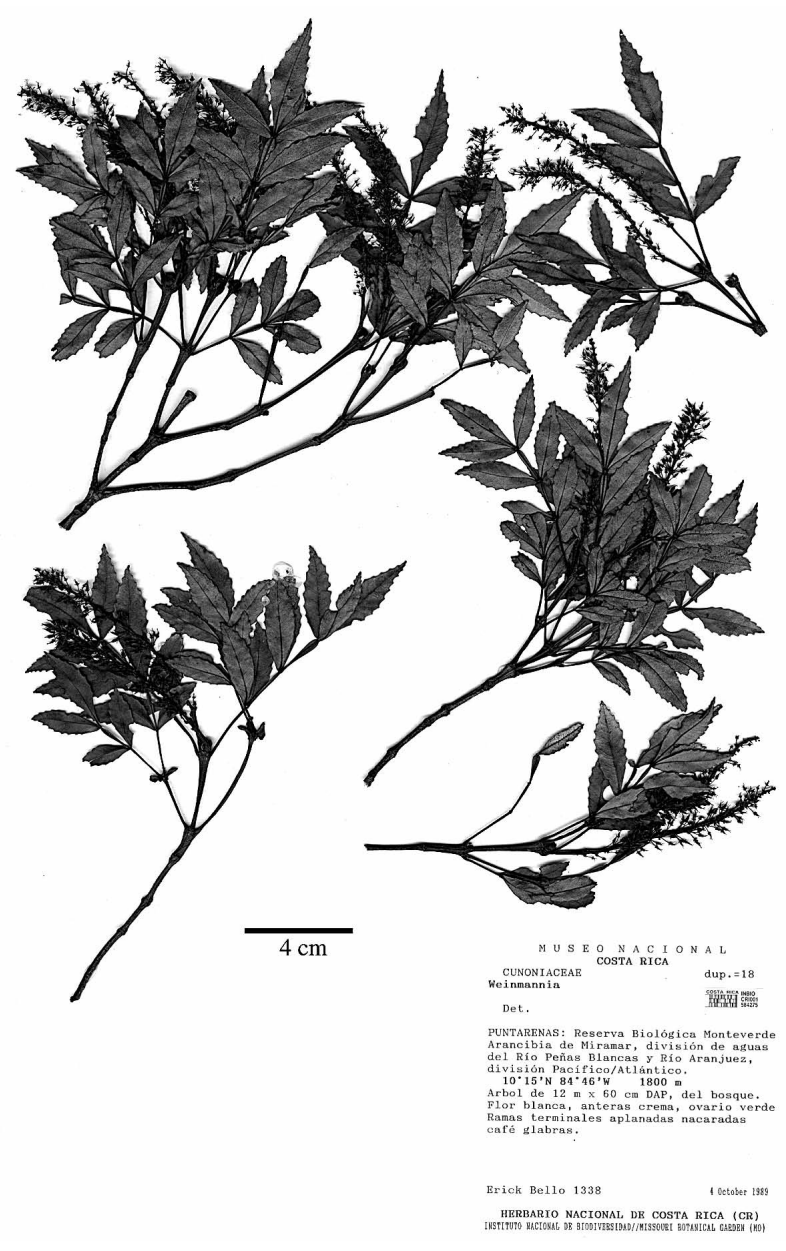

Fig. 4. Weinmannia burserifolia (Bello 1338, INB). 
como W. liebmannii y, aunque parece ser distinta de $W$. burserifolia, no se puede tomar una decisión definitiva basado en el poco material disponible, por lo que se trata como un sinónimo de este último taxon. Estudios posteriores (sobretodo a nivel de poblaciones locales en Oaxaca) pueden probar que se trata de un taxon distinto del concepto de W. burserifolia acá propuesto.

Bernardi (1961) reportó la presencia de W. multijuga Killip \& A.C. Smith en Costa Rica, pero el espécimen citado como testigo $(A$. Smith $155, \mathrm{~F})$ en realidad representa a $W$. burserifolia.

\section{Especímenes examinados}

MÉXICO. Chiapas: N de Jitotol, 28-IX-1971, Breedlove 19958 (F, MO); Tenejapa, Paraje Banabil, 7-XI-1971, Breedlove \& Smith 22029 (MO); Cintalapa, entre Rizo de Oro y Colonia Figaroa, 8-I1973, Breedlove 31418 (F, MEXU). Oaxaca: s.l.,-V-1842, Liebmann 1776 (C [fotografías, INB]); entre Hueytamalco y Tizatlán, V-1841, Liebmann 1777 (C [fotografías, INB]); Amatlán, I-1842, Liebmann 1779 (C [fotografías, INB]).

NICARAGUA. Jinotega: Macizos de Peñas Blancas, 14-I1979, Stevens 11327 (MEXU, MO). Zelaya: Cerro Saslaya, W de Siuna, 5-V-1977, Neill 1844 (MO), 4-V-1978, Neill 3861 (MO).

COSTA RICA. Alajuela: Arancibia de Mirarmar, 4-X-1989, Bello 1338 (CR, INB, MO); reserva biológica Monteverde, río Peñas Blancas, 7-XII-1989, Bello 1560 (CR, INB, MO); reserva biológica Monteverde, Peñas Blancas, 8-XI-1993, Haber E Ramírez 11680 (INB); La Fortuna de San Carlos, Volcán Arenal, 8-XI1989, Jiménez 747 (CR, MEXU, MO); Palmira, Zarcero, 16-VIII1934, A. Smith 155 (CR, F, MO); Parque Nacional Rincón de la Vieja, Laguna Jilgueros, 9-I-1993, Morales \& al. 2244 (CR, INB, MO). Cartago: El Guarco, Ojo de Agua, 17-X-1966, Mora 6 (CR, F); SE del Empalme, 18-VII-1977, Wilbur \& al. 22882 (CR, DUKE, F, MO); El Retiro, Santa Cruz de Turrialba,-I-1941, Valerio 1263 (CR, F). Guanacaste: Parque Nacional Guanacaste, Volcán Cacao, 15-VII-1989, Hammel É al. 17650 (INB, MO). Heredia: Parque Nacional Braulio Carrillo, Cerros Las Marías, 24-V1992, Boyle 854 (CR, INB, MO); Parque Nacional Braulio Carrillo, sendero del transecto, 5-VI-1992, Boyle 920 (MO), 29-VII1992, Boyle \& Hibbs 960 (MEXU, MO), 23-VIII-1992, Boyle E Hibbs 3175 (MO); Parque Nacional Braulio Carrillo, estación Barva, 3-XI-1991, Fernández 152 (CR, INB, MO). Limón: Parque Internacional La Amistad, cerros Dudu y Apri, 21-VII-2000, Alfaro 3279 (INB); Cordillera de Talamanca, entre el río Tararia y macizo Kamuk, 17-IX-1984, Davidse E Herrera 29261 (MO); Cordillera de Talamanca, quebrada Kuisa, 17-III-1993, Fernández 706 (INB, MO). Puntarenas: Cerro Plano, Monteverde, 1-VIII-1992, Bradford 97 (MO). San José: S de la Georgina, 1-VIII-1977, Almeda E al. 3347 (DUKE, F); entre El Empalme y Villa Mills, 28-VIII-1960, Cruz 184 (MO); Parque Nacional Chirripó, sendero a Cerro Urán, 6 -IV-1995, González 632 (INB, MO); Finca Madre Selva, Trinidad de Dota, 5-I-1990, Wiemann 293 (F).

PANAMÁ. Chiriquí: Finca Lerida, quebrada Velo, 25-VII1947, Allen 4736 (F, EAP, G, MO, S, U); entre Alto Quile y Bajo Mono, 10-II-1985, Correa \& al. 4662 (MO, PMA); Cerro Horqueta, 13-VIII-1974, Croat 26930 (MO); faldas Volcán Barú, 18-V1976, Croat 35008 (MEXU, MO); La Popa, 6-VIII-1972, D’Arcy 6446 (MO, U); Barú, Boquete, 20-III-1977, D’Arcy 11058 (MO); faldas del Barú, Alto Pineda, 9 -IV-1979, D’Arcy \& al. 13215 (MO); cerro Horqueta, 1 May, sin año, Hagen \& Hagen 2068 (MO); entre Cerro Punta y Boquete, 16-III-1983, Hamilton $\mathcal{E}$
Stockwell 3362 (MO); Alto Los Guerra, W de Bambito, 13-VII1983, Hamilton E Krager 3879 (MO); Hato de Volcán, 5 -IV-1979, Hammel E al. 6753 (MO); Boquete, entre Bajo Mono y Alto Quiel, 8-II-1986, McPherson E Merello 8363 (MEXU, MO, U); NW de Cerro Punta, estación INRENARE, 18-X-1992, McPherson 15916 (MO); Parque Internacional La Amistad, entre el Cerro Shurivo y el río Terbi, 19-IX-1997, Morales \& Quesada 5817 (INB, MO); faldas del Volcán Barú, 21-IV-1975, Mori \& Kallunki 5692 (MO); cerca de Cerro Punta, 14-V-1984, Murphy 1023 (DUKE, MO); Boquete, camino a Quiel, 13-V-1977, Proctor 31843 (MO); Volcán Barú, 8-III-1983, Schmalzel 1439 (MO); Boquete, Finca Collins, 13-III-1963, Stern E al. 33786 (MO, US); N del Volcán, 28-VI1969, Tyson 5843 (MO); entre Bajo Quiel y Bajo Mono, NW de Boquete, 30-V-1970, Wilbur \& al. 11963 (DUKE, F, MO); Casita Alta, Volcán de Chiriquí, 1938, Woodson E al. 821 (MO).

4. Weinmannia fagaroides Kunth, Nov. Gen. Sp. (quarto ed.) 6: 54, t. 524. 1823

Tipo: Ecuador. Loja: "prope Loxa Peruvianorum", VII (año perdido) (fl), Humboldt E Bonpland s.n. (holótipo, P-HB [fotografía F neg 34635]). Fig. 5.

Arbustos a árboles de 1-10 m de altura, los tallos diminuta y densa o moderadamente puberulentos, glabrescentes o glabros con la edad. Hojas compuestas, con (7)11-17(25) foliolos; lámina 4-8(10) × 2-5(6) $\mathrm{mm}$, elíptica, obovada a obovado-elíptica, membranácea, usualmente glabra, más raramente glabrescente con unos cuantos pelos a lo largo del nervio central en la superficie abaxial, la mayoría con el ápice obtuso a redondeado, la base obtusa a redondeada, los márgenes enteros, subenteros o levemente crenados hacia el ápice, pero pareciendo enteros, no revolutos; entrenudos del raquis alados, las alas de 1,5-3 mm de ancho, el raquis esparcidamente puberulento a glabrescente en la superficie abaxial, usualmente solo con grupos de pelos en los nudos, el indumento del mismo color del raquis; peciolos 2,5-4 mm de largo, usualmente no alados, densa a moderadamente puberulentos, estípulas 5-7 × 4,5-6,5 mm, anchamente ovadas a suborbiculares. Inflorescencias $2,5-7 \mathrm{~cm}$ de largo, el raquis y los pedicelos moderada a esparcidamente puberulentos, el indumento no ferrugíneo ni amarillo, diminuto; flores 2,5-3 $\mathrm{mm}$ de largo, pedicelos 1-3,2 mm de largo, lóbulos del cáliz 0,8-1 mm de largo, glabrescentes, el ápice agudo, pétalos ca. $2 \mathrm{~mm}$ de largo, ovario ca. 1,5 mm de largo, glabro. Cápsulas 3-3,5 mm de largo, glabras.

Distribución, bábitat y ecología. Honduras, Costa Rica hasta Colombia, Venezuela, Ecuador, Perú y Bolivia, creciendo en bosques secundarios, robledales, márgenes de páramos, áreas de vegetación subparamosa, entre los 2200-3300 m. Especímenes con flores $\mathrm{y}$ frutos se han recolectado en forma intermitente a través de todo el año, pero con un pico entre agosto y febrero. 
Weinmannia fagaroides es una distintiva especie común en los bordes de páramo y vegetación de transición a páramo en Centroamérica. Aunque puede intergradarse en algunas áreas con W. pinnata L., se reconoce fácilmente por el usual pequeño tamaño de sus foliolos, con la lámina casi entera y levemente crenada distalmente.

Por lo general, las poblaciones que se encuentran entre los 2700-3000 m, tienden a tener hojas con algunos foliolos hasta de $1 \mathrm{~cm}$ de longitud, pero siempre predominan los foliolos de dimensiones pequeñas. En estados juveniles esta especie usualmente tiene hojas con muchos más foliolos, que en relación a ramos floríferos, pero se puede reconocer por su distribución limitada a zonas subparamosas y tamaño de la lámina foliar. $W$. fagaroides se integra con W. pinnata por debajo de los $2900 \mathrm{~m}$ y probablemente se hibriden, ya que ambas especies crecen simpátricamente.

Las colecciones de Honduras tienden a tener más foliolos (hasta 23) en relación al material del $\mathrm{N}$ de Centroamérica y Sudamérica. Estudios monográficos posteriores podrían determinar que debe tratarse como una especie aparte, pero por ahora preferimos incluir este material dentro del concepto de W. fagaroides manejado aquí.

\section{Especímenes examinados}

HONDURAS. Comayagua: río Acajual, SW de Gracias, 16-V1992, D’Arcy 17955 (EAP, MO). Lempira: al NW de El Súcte, Parque Nacional de Celaque, río Arcágual, 15-V-1992, Mejía 100 (EAP, MO, TEFH); al NW de El Súcte, Parque Nacional de Celaque, 17-II-1993, Mejía 310 (EAP); Montaña de Celaque, valle del río Arcagual, V-1991, Davidse E Zúñiga 34823 (EAP, MO, TEFH); Celaque,-XI-1974, Hazlett 2231 (MO); Celaque, 4-6-VI1978, Hazlett \& Coe 2879 (TEFH); río Arcagual, Parque Nacional de Celaque, 15-XI-1991, Hawkins 72 (EAP, TEFH); El Mojón, 17-V-1992, Hawkins \& al. 353 (EAP, MO, TEFH); cerro Las Minas, montaña de Celaque, Gracias, 20-V-1998, Padgett E al. 266 (EAP).

COSTA RICA. Alajuela: Volcán Poás, 12-VIII-1967, Lent 1197 (F). Cartago: El Guarco, Ojo de Agua, 16 -IV-1999, Alfaro 92 (INB, MO); NE de Villa Mills, 19-VI-1966, Anderson \& Mori 67 (F); Volcán Turrialba, 13-II-1922, Greenman E Greenman 5607 (MO); Villa Mills, 16-I-1987, Hill \& al. 17889 (MO); Volcán Irazú, 26-VI-1949, Holm E Iltis 148 (F, G, P, U); La Chonta, 24-II-1994, Jiménez É al. 1484 (INB, MO); Cerro de la Muerte, 16-VIII-2002, Kriebel 958 (INB, MO); Santa Cruz, Volcán Turrialba, Cerro Tiendilla, 29-III-1994, Lépiz E Morales 272 (CR, INB, MO); Volcán Turrialba, 29-III-1994, Morales É Lépiz 2606 (INB, MO); Fila Ojo de Agua, Sitio Burros, 11-VIII-1994, Morales E Lépiz 3094 (INB, MO); Cerro Vueltas, 14-VIII-1994, Morales E Abarca 3099 (INB, MO); Cerro Jaboncillo, Cordillera de Talamanca, 14VIII-1994, Morales \& Abarca 3105 (INB, MO); SW de Cartago, 12-VIII-1967, Taylor 4344 (MO); río Birris, Volcán Irazú, 30-VII1977, Webster 22129 (F, MO); NW de Villa Mills, 28-III-1967, Wilbur E Stone 8770 (DUKE, F); Volcán Irazú, 1948, Williams E Molina 13917 (EAP, F, MO); Cerro de La Muerte, 6-II-1963, Williams E al. 24456 (EAP, F); Cerro Sábira, Macizo Buena Vista, 5 -
VI-1993, Vargas \& al. 1415 (CR, MO). Heredia: Parque Nacional Braulio Carrillo, Volcán Barva, 16-III-1992, Boyle 745 (MO); Parque Nacional Braulio Carrillo, Laguna Barva, 13-VIII-1993, Boyle E al. 2358 (MO), Boyle \& al. 2359 (CR, MO), Boyle \& al. 2363 (CR, MO), Boyle E al. 2367 (CR, MO), Boyle E al. 2368 (CR, MO); 14-VIII-1993, Boyle \& al. 2384 (MO); Parque Nacional Braulio Carrillo, Volcán Barva, 13-VIII-1993, Bradford E al. 2363 (MO); Parque Nacional Braulio Carrillo, Barva, 15-III-1995, Fernández 1541 (INB, MO); laguna del volcán Barva, 6-II-1890, Tonduz 1950 (BR, G). Limón: entre Cerro Kasir y Cerro Nai, 22III-1984, Davidse \& al. 25856 (F, MO); cerro Kamuk, 17-18-IX1984, Davidse E Herrera 29309 (MO). Puntarenas: Parque La Amistad, sendero Herradura-Urán, 23-II-1995, Aguilar \& al. 3933 (INB, MO); Herradura-Urán, 8 -IV-1995, Aguilar E Garrote 3996 (INB, MO); Parque Internacional La Amistad, Tararia, cerro Dudu, 1-I-1997, Quesada E Sancho 1819 (INB); Parque Internacional La Amistad, cerro Echandi, 13-VIII-1997, Quesada E al. 1992 (INB). San José: Los Chespiritos, 23 -IV-1992, Aguilar 1181 (INB, MO); O de la Georgina, 8-III-1981, Almeda E Nakai 4808 (CAS, CR); Cerro de la Muerte, 15-VII-1988, Boyle \& al. 5437 (CR); La Georgina, 4-VIII-1965, Croat 325 (MO); Cerros Cuerecí, 18-IX-1983, Davidse 24864 (MO); Cerro de la Muerte, 4 -IV-1978, Davidson 7246 (F); Parque Nacional Chirripó, sendero a cerro Urán, 30 -IV-1997, Gamboa E al. 1249 (INB, MO); Reserva Forestal río Macho, Estación Ojo de Agua, 24-VI-1997, Gamboa 1537 (CR, INB, MO); Cerro Urán, 7 -IV-1995, González 655 (INB, MO); Cerro de la Muerte, Villa Mills, 17-III-1997, Hammel E González 20884 (INB, MO); Chirripó Grande, 1932, Kupper 1310 (M); Cerro de la Muerte, 13-VIII-1993, Morales E al. 1609 (INB,
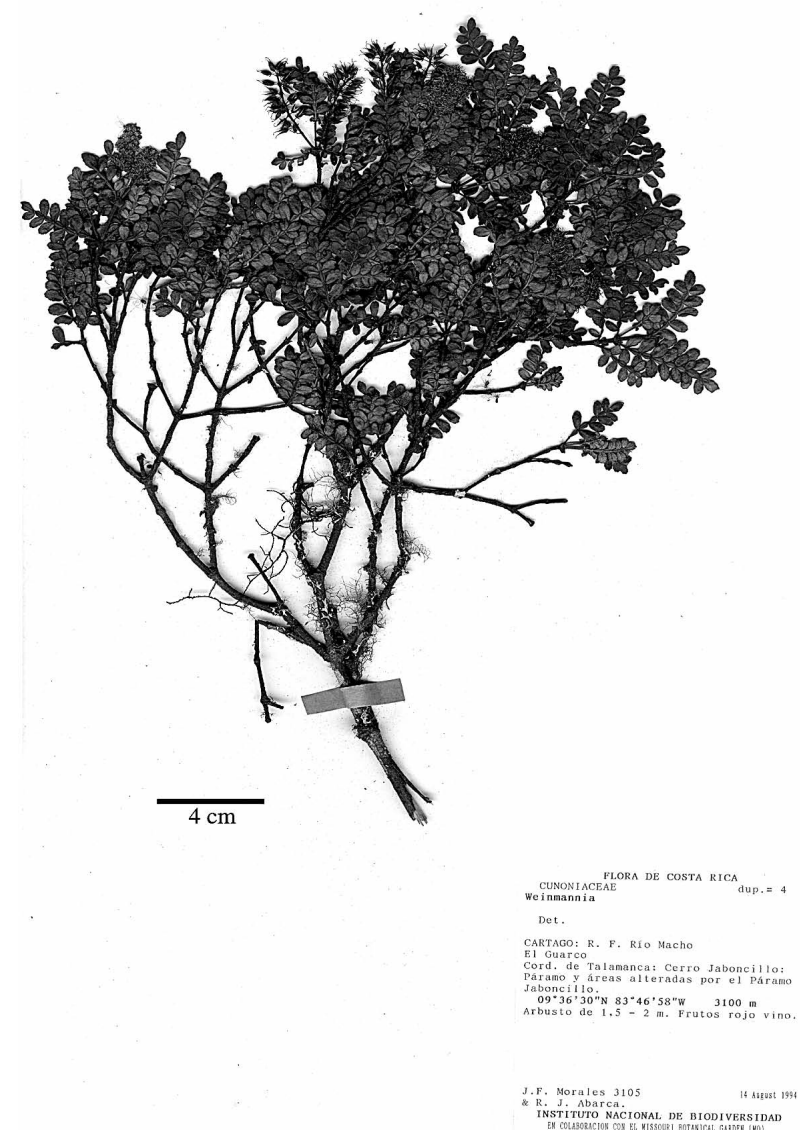

Fig. 5. Weinmannia fagaroides (Morales \& Abarca 3105, INB). 
MO); Villa Mills, 6-IX-1996, Ramírez 454 (INB, MO); Ojo de Agua, 2-I-1990, Wiemann 279 (F), Wiemann 280 (F).

PANAMÁ. Bocas del Toro: río Colubre,-VIII-1983, Gómez E al. 21876 (MO). Chiriquí: El Volcán, SE de la Nivera, 15-III-1979, D'Arcy \& Hammel 12501 (MO).

\section{Weinmannia horrida J.F. Morales, sp. nov.}

Tipo: Costa Rica. Puntarenas: Cantón de Coto Brus, Las Mellizas, siguiendo la línea divisoria Costa Rica-Panamá, entre Cerro Pando y Cerro Nubes, camino de la Sierra, 2465 m, 15-VIII-1989 (fl, fr), Chacón 328 (holótipo, INB; isótipo, MO). Fig. 6.

$A$ Weinmannia tomentosa L.f. affine, foliolis [8]10$17 \times[5] 6-8 \mathrm{~mm}$ (vs. 3-9 $\times 2-4,5 \mathrm{~mm})$, subtus sparse birsútulis et margine revolutioribus differt.

Arbustos de 3-7 m de altura, los tallos densamente hirsutos, el indumento amarillo, con la edad esparcidamente puberulentos o glabrescentes. Hojas compuestas, con 7-13(15) foliolos; lámina (8-)10-17 × (5)6-8 mm, elíptica, membranácea, variadamente hirsuta o hirsútula abaxialmente, algunas veces la lámina con el indumento muy esparcido y restringido a las ve-

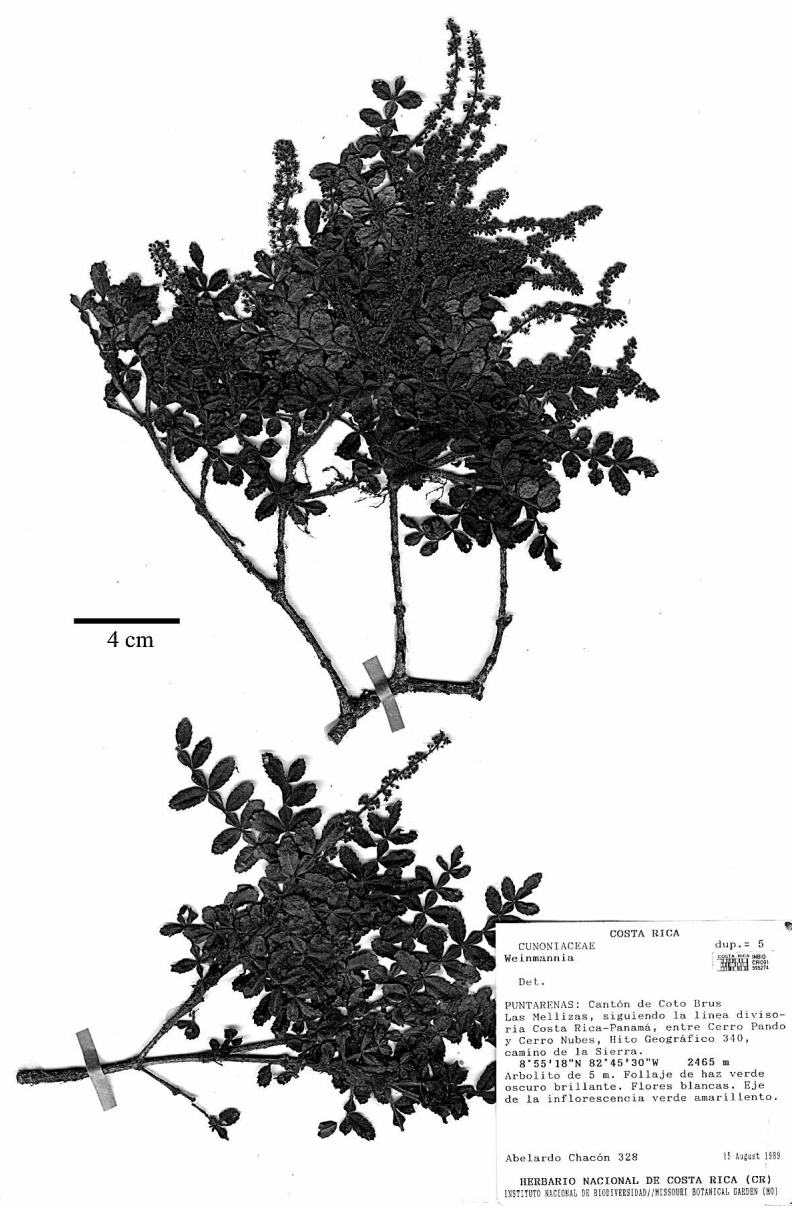

Fig. 6. Weinmannia horrida (Chacón 328, INB). nas, el indumento más esparcido en la superficie adaxial, el ápice redondeado, obtuso o agudo, la base obtusa o anchamente aguda, los márgenes serrados, no revolutos; entrenudos del raquis alados, las alas 3-5 $\mathrm{mm}$ de ancho, el raquis densamente hirsuto o hirsútulo abaxialmente, el indumento amarillo; peciolos 5-6 mm de largo, densamente hirsutos, no alados o angostamente alados distalmente; estípulas 6-7 $\times$ 6-7 $\mathrm{mm}$, suborbiculares. Inflorescencias $5-8 \mathrm{~cm}$ de largo, el raquis y los pedicelos densamente hirsutos, el indumento amarillo, conspicuo; flores ca. $2 \mathrm{~mm}$ de largo, pedicelos 1,5-3 mm de largo, lóbulos del cáliz 1$1,2 \mathrm{~mm}$ de largo, esparcidamente hirsútulos, el ápice agudo, pétalos ca. 1,5 mm de largo, ovario ca. 1,5 mm de largo, glabro. Cápsulas 2-2,5 mm de largo, glabras o glabrescentes.

Distribución, hábitat y ecología. Restringida por el momento al S de Costa Rica y NW de Panamá, en la Cordillera de Talamanca, donde crece en robledales entre los 2400-2700 m de elevación. Especímenes con flores fueron recolectados en junio, agosto y septiembre. Frutos se han recolectado en septiembre.

Weinmannia horrida es similar a $W$. tomentosa L.f., pero esta especie se separa por sus foliolos conspicuamente revolutos (vs. planos) y tomentosos (vs. esparcidamente hirsútulos) abaxialmente. Además, los foliolos de $W$. horrida son usualmente más grandes [(8)10-17 × (5)6-8 mm] que los de W. tomentosa (3-9 $\times 2-4,5 \mathrm{~mm})$. Otras especies relacionadas incluyen W. mariquitae Szyszyl. y W. haenkeana Engl., pero la primera especie tiene frutos mucho más grandes, mientras que en la segunda los foliolos son más desarrollados y las inflorescencias mucho más largas. Otras especies relacionadas en Sudamérica incluyen a W. cinerea Ruiz \& Pav., pero esta especie difiere por su indumento, mucho más corto y de color negro o negruzco, así como por sus foliolos que tienden a tener los márgenes algo revolutos; y también $W$. reticulata, que se reconoce por sus hojas con foliolos mucho más pequeños. Weinmannia pinnata se distingue de esta nueva especie por sus tallos densa a moderadamente puberulentos (no hirsutos), glabrescentes con la edad, foliolos con la lámina glabra o glabrescente y puberulentos a lo largo del nervio central abaxialmente (vs. variadamente hirsutos o hirsútulos), así como inflorescencias con el raquis y pedicelos densamente hirsutos (vs. esparcida a moderadamente puberulentos o puberulento-hirsútulos en las Antillas Mayores). Weinmannia pinnata es una especie polimórfica, con una gran variedad de formas encontradas a través de todo el rango de distribución geográfica, pero W. borrida tiene suficientes caracteres para considerla como un taxon aparte. Probablemente, los sitios donde se 
encuentra la más alta diversidad de W. pinnata, tanto en forma como en pubescencia correspondan a las Antillas Mayores, y aunque ocasionalmente también existen especímenes que tienen tallos puberulentohirsútulos de esas zonas geográficas, la pubescencia nunca es consistentemente hirsuta y distribuida prácticamente en todas las partes de la planta como en W. borrida.

\section{Especímenes examinados}

COSTA RICA. Limón: Parque Internacional La Amistad, Tararia, Valle del Silencio, 22-VI-2003, Alfaro 4654 (INB, MO); Parque Internacional La Amistad, Cerro Asidbeta, 6-VI-1996, Moraga 384 (INB, MO).

PANAMÁ. Chiriquí: Renacimiento, Jurutungo, Cerro Pando, 22-IX-1996, Aranda 3100 (MO)

6 Weinmannia intermedia Schltdl. \& Cham., Linnaea $5: 555.1830$

Tipo: México. Veracruz: Cuesta Grande, entre Jalacingo y Huittamalco, XII (año perdido) (fl), Schiede E Deppe 521 (lectótipo, designado por Bernardi (1961), B; isótipos, BR, NY, P, U). Fig. 7.

Árboles a arbustos de 3-15 m de altura, los tallos densamente tomentulosos o puberulentos, glabrescentes con la edad, el indumento algo amarillento o algunas veces del mismo color de las ramitas. Hojas compuestas, con (11)13-21(25) foliolos; lámina (5)8$16(20) \times 3-8(11) \mathrm{mm}$, usualmente elíptica, membranácea, la superficie abaxial densa a moderadamente puberulenta a glabrescente, sobretodo a lo largo de los nervios (el indumento no amarillo ni hirsuto o hirsútulo), la superficie adaxial glabrescente, la mayoría con el ápice obtuso a redondeado, la base cuneada a obtusa, los márgenes serrados o crenado-serrados a lo largo de casi toda su longitud, no revolutos; entrenudos del raquis alados, las alas de $1,5-5 \mathrm{~mm}$ de ancho, el raquis densa a moderadamente puberulento abaxialmente, con grupos de pelos en los nudos, el indumento del mismo color de la lámina al secar; peciolos 5-9 $\mathrm{mm}$ de largo, densamente tomentulosos, usualmente no alados; estípulas $5-7 \times 4-7 \mathrm{~mm}$, suborbiculares a ampliamente ovadas. Inflorescencias 4-12 $\mathrm{cm}$ de largo, el raquis y los pedicelos densamente puberulentos, el indumento no amarillo ni hirsuto, adpreso y diminuto; flores de 1,5-2 mm de largo, pedicelos 1,5-3,7 mm de largo, lóbulos del cáliz ca. $1 \mathrm{~mm}$ de largo, glabrescentes, el ápice agudo, pétalos ca. 2,2 $\mathrm{mm}$ de largo, ovario ca. 1,5 mm de largo, glabrescente. Cápsulas 2-2,5 mm de largo, glabras.

Distribución, hábitat y ecología. Endémica de México, donde crece en bosques húmedos y ventosos entre los 1300-2550 m. Especímenes con flores han sido recolectados entre febrero y octubre. Frutos se han recolectado en abril, junio, julio y diciembre.

Weinmannia intermedia puede ser confundida en Centroamérica con la común $W$.pinnata L., pero esta última especie tiene hojas usualmente con menos foliolos [5-11(15) vs. (7)13-21(25)], con las láminas usualmente mucho más pequeñas, así como frutos más grandes (3-3,5 vs. 2-2,5 mm). En forma general, es frecuente que los especímenes posean un mayor número de foliolos, en relación al típico patrón de $W$. pinnata encontrado en el resto de Centroamérica.

\section{Especímenes examinados}

MÉXICO. Hidalgo: NE de Agua Blanca, Iturbide, 22-VII1973, Gimate 1077 (EAP, ENCB, WIS); W de Tenango de Doria, 7-VII-1979, Hernández 3435 (MEXU, MO); Barranca, Trinidad, 28-VII-1904, Pringle 8977 (C, FI, G, M, MO, NY, P, W, Z), 21VIII-1905, Pringle 10026 (C, F, FI, G, M, MO, P, W). Oaxaca: Ixtlán, Comaltepec, 9-X-1991, Boyle E Boyle 486 (MO); Comaltepec, entre Ixtlán de Juárez y Valle Nacional, 12-X-1991, Boyle E Boyle 511 (MO); Ixtlán, Comaltepec, cerca de Vista Hermosa, 16XI-1991, Boyle E Boyle 639 (MO); Ixtlán, entre Tuxtepec y Oaxaca, 7-IV-1981, Cedillo E Lorence 672 (F, MEXU, MO); SW de Talea, Villa Alta, 19-IV-1982, Cedillo E Lorence 1266 (MEXU, MO); NE de Huautla, camino a Teotitlan, 18-VI-1982, Cedillo E al. 1644

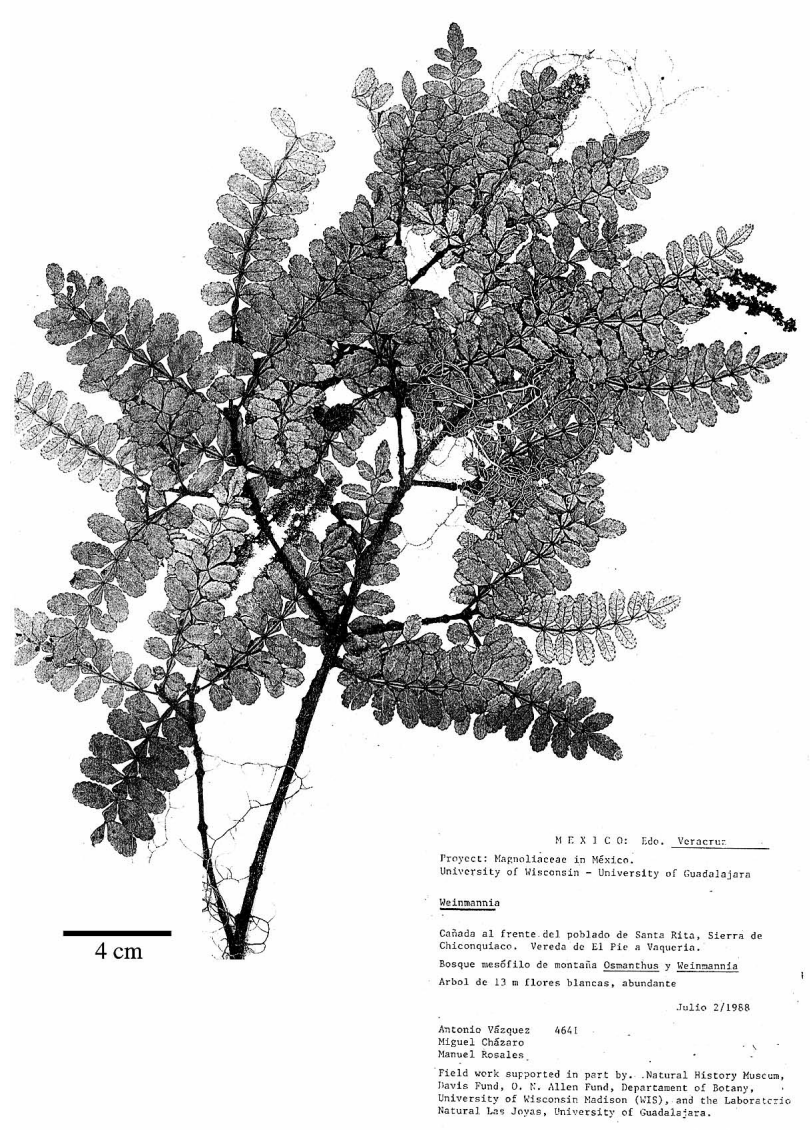

Fig. 7. Weinmannia intermedia (Vásquez et al. 4641, INB). 
(MEXU, MO); Monte Pelado, VII (año perdido), Galeotti 2838 (BR, G, P, W); Mixistlán, S de Totontepec, 10-XI-1983, García E al. 1301 (MEXU, MO); cerro Pelón, carretera Oaxaca-Tuxtepec, 4-VIII-1985 (fl), García \& al. 1843 (BIGU, MEXU); río Soyolape, 4-VIII-1975, Lawton 792 (F); Ixtlán, Comaltepec, La Esperanza, 20-IX-1988, López E Martin 371 (MO); Ixtlan, Comaltepec, 7-IV1981, Martin 499 (MO); Mixe, Tontepec, vía Ayutla a Totontepec, 18-XII-1985, Nee E Martin 32261 (MO); Ixtlán, N del Mirador y de Humo Chico, 26-VIII-1986, Torres E Cortés 8686 (EAP, MEXU, MO); Sierra Madre Oriental, NE de Cerro Pelón, 19-VII1972, Webster \& al. 17462 (MO, U, UC). Puebla: cerca de Xalacapa, 18-X-1945, Hernández E Sharp 96 (MEXU, MO). Veracruz: Soteapan, Sierra de Santa Marta, Ocotal Chico, 22-VI-1972, Beaman 6226 (F); Acajete, Plan de Cedeño, 13-III-1979, Calzada 5240 (F); Acajete, Ranchería El Rincón, 16-VII-1979, Calzada 5425 (F); Alto Lucero, Cerro de La Cima, 10 -IV-1981, Castillo \& Vásquez 1536 (MO); Altotongal, Barranca de Tatempan,-VI-1980, Cházaro 1188 (F); Chiconquiaco, Planta de Pie, VII-1991, Cházaro E Narave 1680 (F); Chiconguiaco, El Jorobado, 11-VII-1984, Cházaro E Robles 3109 (WIS); Ayahualulco, entre Carrizal y Cerro Boludo, 18-VIII-1985, Chazaro \& Robles 3644 (WIS); Misantla, carretera Xalapa-Veracruz, 2-V-1972, Dorantes 588 (BR, F, MEXU, MO); Huayacocotla, borde con Hidalgo, 13-VII-1977, Fay \& Calzada 876 (F, MEXU); Huayacocotla, Viborillas, 10-II-1972, Hernández 1463 (F, MEXU); Huayacocotla, camino a Rancho Nuevo, 11-II-1972, Hernández 1473 (F, MEXU); La Cima, Plan de Las Hayas, 21-VI1972, Hernández 1557 (F); La Calavera, Atzalan, 17-V-1973, Márquez \& Dorantes 114 (F, MEXU, MO); Plan de Sedeño, Banderilla, 6-II-1976, Márquez 486 (F, MO); La Calavera, N de Altotonga, 28VI-1980 (fr), Nee E Hansen 18662 (MO); entre Huayacocotla y Zontecomatlán, NE de Agua de La Calabaza, 27-IV-1983, Nee E Taylor 26901 (F, XAL); Naolinco,VII.1912, Purpus 5931 (MO); NNE de Huayacocotla, camino a Zontecomatlán, 27-I-1984, Taylor \& Nee 265 (F, MO, XAL); Huayacocotla, entre Helechales y Los Ocotes, 21-III-1980, Vargas 209 (MO); carretera Huayacocotla-Zilacatipán, 7-VII-1980, Vargas 349 (MEXU, MO); Sierra de Chiconquiaco, entre El Pie y Vaquaria, 2-VII-1988, Vásquez E al. 4641 (F, WIS); Atzatlan, Alzezeca, 16-III-1970, Ventura 1089 (ENCB, MO); Acajetes, Plan de Sedeño, 10-VI-1971, Ventura 3676 (F); El Huérfano, Chiconquiaco, 10-VII-1973, Ventura 8582 (EAP, ENCB, P, WIS); El Huérfano, Chiconquiaco, 13-IX-1973 (fr), Ventura 8968 (EAP); Plan de Cedeño, Acajete, 23-VI-1976, Ventura 12885 (WIS); La Chiveria, Noalinco, 18-VI-1983, Ventura 20318 (MO). Estado desconocido: Monte Pelado, 184? (fl), Galeotti 2838 (W). Datos perdidos: Sessé E Mociño 991 (F, MA).

7. Weinmannia karsteniana Szyszyl., Oesterr. Bot. Z. 40(2): 41.1890

Tipo: Venezuela. Mérida: Mérida, fecha perdida (fl), Karsten s.n. (holótipo, W [fotografía F neg. 30156]). Fig. 8.

Weinmannia myrtifolia Cuatrec., Caldasia 1(2): 16, f. 4, 5B. 1941

Tipo: Colombia. Cundinamarca: Cordillera Oriental, Páramo de Guasca, Juiquin, quebrada Amarilla, 2840 m, 2-VI-1940 (fl), Cuatrecasas 9451 (holótipo, COL; isótipos, F [2 pliegos, fotos F neg. 39826 y 39827], U, US [2 pliegos]).

Árboles o arbustos de 5-10 m de altura, los tallos jóvenes densa a esparcidamente puberulentas, el indu- mento no ferrugíneo ni amarillento, glabrescentes o glabros con la edad. Hojas simples; lámina 9-18(22) × 7-14(16) mm, suborbicular, subcoriácea, glabra o glabrescente e inconspicuamente puberulenta a lo largo del nervio central en la superficie abaxial, el ápice y la base redondeada a obtusa, los márgenes crenados en los 3/4 o mitad distal, enteros cerca de la base, no revolutos; peciolos hasta $2 \mathrm{~mm}$ de largo, no alados, glabros o glabrescentes; estípulas inmaduras 3-5 × 2 $3,5 \mathrm{~mm}$, suborbiculares. Inflorescencias $2-6 \mathrm{~cm}$ de largo, el raquis y los pedicelos moderada a esparcidamente puberulentos, el indumento no ferrugíneo ni amarillento, diminuto; flores 2-2,5 mm de largo, pedicelos 1,5-3(-3,5) mm de largo, lóbulos del cáliz ca. 1 $\mathrm{mm}$ de largo, glabros o glabrescentes, el ápice agudo, pétalos ca. 1,5 mm de largo, ovario 1,5-1,7 mm de largo, tomentuloso. Cápsulas de 2-3 mm de largo, densamente puberulentas, de manera inusual glabrescentes con la edad.

Distribución, hábitat y ecología. Conocida en la Cordillera de Talamanca en Costa Rica, hasta Colombia y Venezuela, donde crece en bosques muy húmedos, áreas alteradas y robledales, entre los 2300-3000 m.
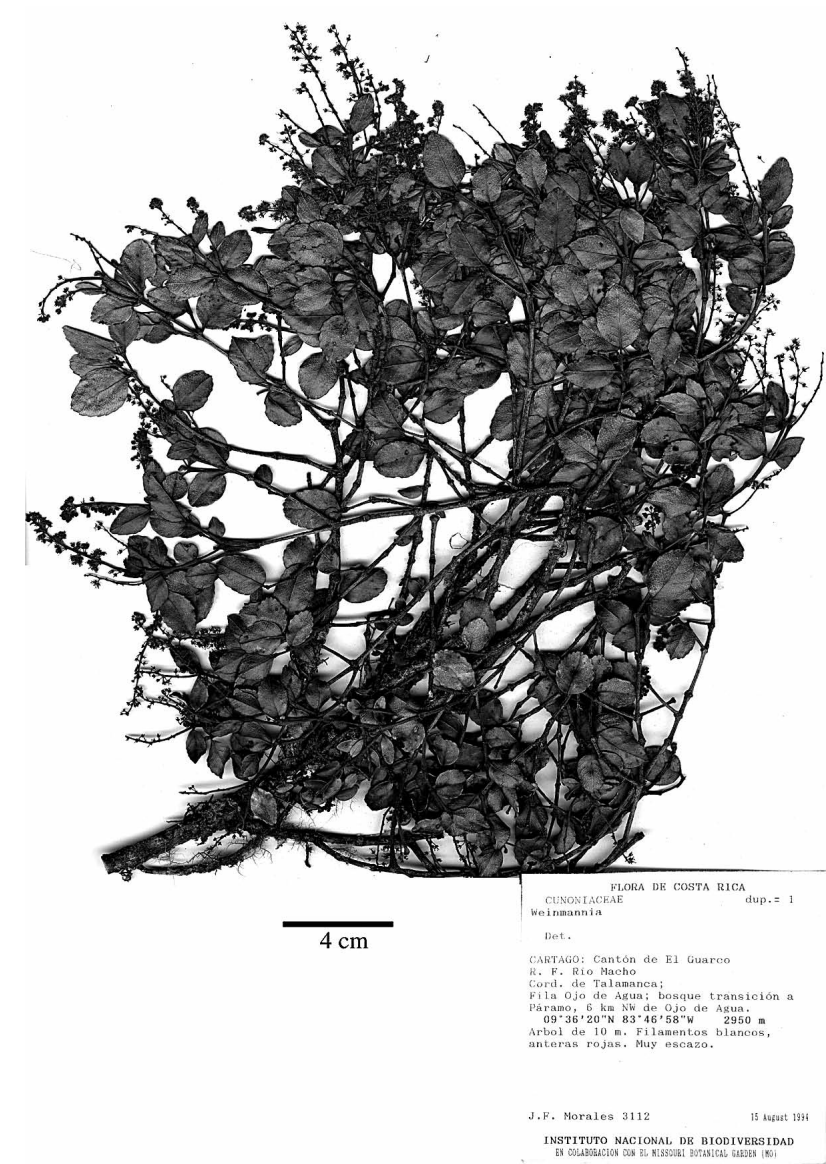

Fig. 8. Weinmannia karsteniana (Morales 3112, INB). 
Floración y fructificación ocurren entre noviembre y agosto.

Weinmannia karsteniana se puede reconocer con facilidad del resto de especies con hojas simples en el $\mathrm{S}$ de Centroamérica por la dimensión de la lámina foliar, que usualmente no excede los 2,2 cm de largo.

\section{Especímenes examinados}

COSTA RICA. Cartago: La Esperanza del Guarco, 21-IV1999, Alfaro E Alfaro 2253 (INB, MO); El Empalme, 9-III-1996, Estrada 464 (CR, INB, MO); Tres de Junio, 28-I-1981, GómezLaurito 6364 (CR); Esperanza del Guarco,-XII-1985, Kappelle 320 (CR); Reserva Forestal río Macho, Finca La Esperanza, 28-VI1994, Lépiz E Ramírez 393 (CR, INB); El Empalme, 2-IX-1966, Madriz 11 (CR); Guarco, Empalme, entrada río Chorizo, 14 -IV1967, Madriz 59 (CR, F); Fila Ojo de Agua, 15-VIII-1994, Morales 3112 (INB); Cascajal, 10-XII-1975, Utley \& Utley 3596 (CR, DUKE, F); NW de Ojo de Agua, 19-III-1978, Wilbur 26067 (DUKE, F, MO). San José: E de la Trinidad, 3-III-1992, Almeda \& Daniel 7211 (CAS, CR, MO); Cienega, Tres de Junio, 15-VIII1960, Cruz 137 (CR, F); Cerro de La Muerte, 5-IX-1973, Poveda 625 (CR); SW del Empalme, 2-II-1970, Wilbur \& Teeri 13911 (DUKE, F, MO).

8. Weinmannia pinnata L., Syst. Nat. (ed. 10) 2: 1005. 1759. Weinmannia glabra L.f., Suppl. Pl. 228. 1781, nom. illeg.

Tipo: Jamaica. Sin datos (fl), Brown s.n. (LINN 508.1 (lectótipo, designado por Harling (1999), fotografías INB). Fig. 9.

Weinmannia intermedia Schltdl. \& Cham. var. pittieri Pamp., Ann. Bot. (Rome) 2: 74. 1905

Tipo: Costa Rica. San José: Cerros de Escazú, 2000 m, XII-1898 (fl), Pittier 13045 (holótipo, G; isótipos, BR, US).

Árboles o arbustos de 2-6 m de altura, los tallos densa a moderadamente puberulentos, el indumento más o menos adpreso (en Centroamérica), no amarillento ni ferrugíneo, glabrescentes con la edad. Hojas compuestas, con 5-11(17) foliolos; lámina 8-18(21) × 4-11 $\mathrm{mm}$, elíptica a obovado-elíptica, membranácea, usualmente glabra o glabrescente y puberulenta a lo largo del nervio central en la superficie abaxial, el ápice usualmente obtuso a redondeado, más raramente agudo o cortamente apiculado, la base obtusa o anchamente cuneada, los márgenes serrados, crenado-serrados o crenados, no revolutos (en Centroamérica); entrenudos del raquis alados, las alas 3-8 $\mathrm{mm}$ de ancho, el raquis glabrescente abaxialmente, con grupos de pelos en los nudos; peciolos 6-11 mm de largo, glabrescentes a densamente puberulentos, no alados o muy angostamente alados distalmente; estípulas 7,5-9,5 × 6,5-9 $\mathrm{mm}$, suborbiculares. Inflorescencias $(3) 6-12(13,5) \mathrm{cm}$ de largo, el raquis y los pedicelos de esparcida a mode- radamente puberulentos (a veces levemente hirsútulos en las Antillas Mayores), el indumento incoloro, diminuto; flores 2-2,5 mm de largo, pedicelos 2-5 $\mathrm{mm}$ de largo, lóbulos del cáliz 0,8-1 mm de largo, glabros o glabrescentes, el ápice agudo, pétalos ca. $2 \mathrm{~mm}$ de largo, ovario ca. 1,5 mm de largo, glabro. Cápsulas 3-3,5 mm de largo, usualmente glabras.

Distribución, bábitat y ecología. Conocida desde el $\mathrm{S}$ de México a través de Centroamérica (excepto El Salvador y Belice) hasta Venezuela, Bolivia y las Antillas Mayores, donde crece en bosques muy húmedos hasta áreas subparamosas, en elevaciones de 1900$3000 \mathrm{~m}$. Especímenes con flores y frutos han sido recolectados en forma irregular durante todo el año.

Weinmannia pinnata es una especie polimórfica, con una gran variedad de formas en relación al numero, tamaño y cantidad de foliolos, lo que hace su taxonomía compleja y difícil. Numerosas poblaciones que se encuentran en Centro y Sudamérica, al ser examinadas en forma aislada parecen representar entidades distinguibles; pero al ser tratadas en un conjunto, la diversa intergradación de carácteres y formas impiden su reconocimiento a nivel específico o infraespecífico. Varios de los especímenes de Guatemala, Honduras y Costa Rica identificados por Bernardi en especímenes de herbario como Weinmannia glabra o W. intermedia en realidad corresponden a esta especie. Casi todas las colecciones de Guatemala examinadas están estériles

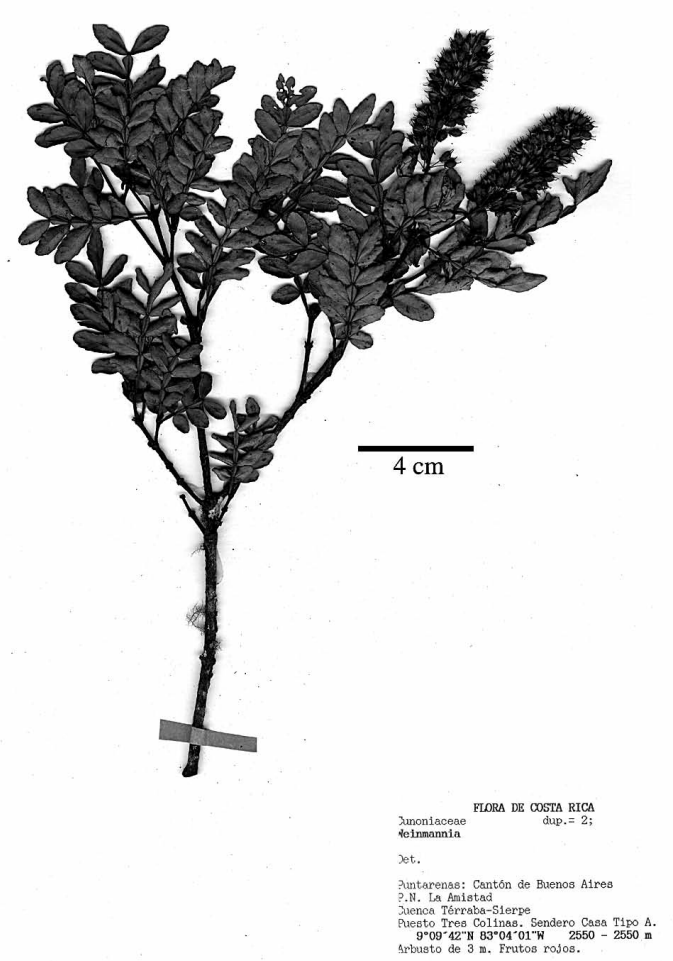

Fig. 9. Weinmannia pinnata (Gamboa \& Picado 582, INB). 
y la inclusión en esta especie está basada en los caracteres morfológicos de las hojas. Sin embargo, debe tenerse cuidado con especímenes estériles, ya que en algunos casos las hojas pertenecientes a brotes tiernos, pueden tener hasta 17 ó 19 foliolos (Márquez E Dorantes $114, \mathrm{~F})$, lo que puede llevar a una identificación errónea, tal y como fue mencionado en la introducción. Para la definición del concepto de $W$. pinnata, descrita originalmente de las Antillas, se ha seguido estrictamente el espécimen tipo, el cual está conformado por un espécimen fragmentario pero representativo, conformado por 3 hojas (una casi incompleta) y una inflorescencia. En este espécimen, las dos hojas preservadas casi completamente tienen 9 foliolos, la lámina con un ámbito de (4)7-9 × (3,2)5-7 mm aproximadamente y con el raquis evidentemente alado. En general y basado en las colecciones examinadas es común que en esta especie las hojas presenten 5-11 foliolos, aunque en especímenes estériles algunos pueden llegar a tener hasta 19. Preferí seguir un criterio más conservador que el utilizado por otros autores (p.ej., Britton, 1905), donde prácticamente sólo se ha reconocido una especie de hojas compuestas en Mesoamérica. De cualquier forma, siempre se encontrarán especímenes con caracteres morfológicos que dificulten su identificación, los cuales probablemente representen híbridos, tal y como se ha reportado en otros países (Harling, 1999).

Un carácter constante a través de las colecciones son los foliolos con el ápice obtuso a redondeado, así como la lámina usualmente glabra en ambas caras (aunque con el raquis y nervio central pubescentes y raramente esparcidamente puberulentas abaxialmente, lo cual se observa en especímenes de las Antillas). El concepto de esta especie manejado por Harling (1999) es mucho más amplio e incluye especímenes con foliolos de dimensiones bastante grandes, algunos de los cuales representan otros táxones como W. lentiscifolia C. Presl. En todo caso y basándose en el tipo de W. pinnata, así como las variaciones encontradas en la localidad tipo (Jamaica) y otros países de las Antillas Mayores (p.ej., República Dominicana), el concepto de esta especie debe estar limitado a los especímenes de hojas más pequeñas.

\section{Especímenes examinados}

MÉXICO. Chiapas: vía San Cristóbal Las Casas a Tenejapa, Manzanita, 30-IX-1965, Breedlove 12440 (MO); San Cristóbal Las Casas, Chamula, paraje Las Ollas, 10-X-1965, Breedlove E Raven 12886 (F); Saxchanal, Sierra Madre, 1-VII-1941, Matuda 4285 (MEXU, MO); Boquerón, Motozintla, 3-V-1945, Matuda 5371 (MO); Santa Rosa, Escuintla, 3-VI-1948, Matuda 17851 (F); Cerro del Boquerón,-VIII-1913, Purpus 6975 (F, MO); Motozintla, entre Ejido Boquerón y Cerro Boquerón, 9-II-1990, Stafford E al. 339 (BM, MO). Oaxaca: Tepitongo, fecha perdida, Galeotti 2837 (BR,
P); San Miguel Chimalapa, Cerro Salomón, 22-XII-1985, Ishiki 1296 (CHAPA, MO); Mixe, río Toro, S de Totontepec, 4-IX1986, E. Ramírez E Ramírez 425 (F, MEXU, MO); Tontontepec, 12-X-1988, Rivera \& Martin 1159 (MO); Totontepec, Mixe, río Toro, 19-IX-1988, Torres E Martínez 12532 (MEXU, MO).

GUATEMALA. Alta Verapaz: San Juan Chamelco, -II-1969, Wilson 40790 (F). Baja Verapaz: Niño Perdido, río La Rinconada, 10-VI-1977, Lundell E Contreras 21974 (MO). San Marcos: Barranco Eminencia, 14-III-1939, Standley 68536 (F); Barranco Eminencia, entre San Marcos y San Rafael Pie de la Cuesta, 6-II-1941, Standley 86317 (F), Standley 86446 (F); Cerro Tumbador, Sierra Madre, 15-XII-1962, Williams E al. 23064 (EAP, F); Sierra Madre, entre San Rafael Pie de la Cuesta y Palo Gordo, XII-1963, Williams E al. 25658 (EAP, F). Zacapa: faldas del Volcán Gemelos, 26-I-1942, Steyermark 43297 (F).

HONDURAS. Morazán: Rancho Quemado, -VI-1948, Molina 1018 (EAP, F, MO); Montaña La Tigra, SW de San Juancito, 11VI-1963, Molina 12735 (EAP, F); San Juancito, 21-V-1947, Williams \& Molina 12800 (EAP, F, MO).

NICARAGUA. Rivas: Volcán Maderas, Balgüe, Isla Ometepe, 14-IX-1983, Nee E Robleto 28099 (MO); Volcán Maderas, 24-II1978, Neill \& Vincelli 3305 (MO); Volcán Maderas, 1-V-1984, Robleto 497 (MEXU, MO); Isla de Ometepe, Volcán Maderas, 24-II1978, Stevens 6498 (MO, TEFH).

COSTA RICA. Alajuela: La Palma, cerros San Antonio, 12VIII-1927, Brenes 5665 (CR); Monteverde, 14-VII-1977, Dryer 1565 (CR, F, MO); Zarcero, Palmira, 30-VIII-1937, A. Smith 259 (F), 26-VI-1937, A. Smith 4219 (F, MO). Cartago: Volcán Turrialba, 3-III-1957, Carlson 3619 (F); La Georgina, 11-III-1987, Grayum \& Affolter 8168 (INB, MO); La Trinidad, 26-II-1966, Molina E al. 17848 (EAP, F); Reserva Forestal río Macho, Salsipuedes, 28 III-1994, Morales 2589 (INB, MO); Cañón, 10 -IV-1994, Morales E al. 2673 (CR, INB, MO); La Georgina, 27-I-1968, Wilbur \& Stone 10056 (DUKE, F, MO); El Retiro, Santa Cruz, I.1941, Valerio 1364 (F); Volcán Turrialba, Trinidad, 25-XII-1974, Wilbur \& Luteyn 18804 (CR, DUKE, F, MO); El Cañón, Cartago, 26-I-1965, Williams \& al. 28210 (C, EAP, F). Heredia: Parque Nacional Braulio Carrillo, Volcán Barva, 16-III-1992, Boyle 756 (MO); entre río Las Vueltas y río Patria, 25-X-1975, Burger E Baker 9496 (CR, EAP, F, U); Estación Barva, 20-I-1994, Fernández 1459 (INB, MO); Porrosatí, Volcán Barva, 11-III-1973, Hartshorn 1150 (CR, F, MO); Monte La Cruz, 30-X-1949, León 283 (CR, F); Volcán Barva, 9-XI1989, Rivera 155 (CR, INB, MO), Rivera 163 (CR, INB, MO); Vara Blanca de Sarapiquí, VII-IX-1937, Skutch 3299 (MO, S, US); entre San Rafael y río Vueltas, 4-IX-1979, Stevens 14056 (F, MO); Barva, 13-VII-1990, Varela 233 (CR, INB, MO); Volcán Barva, 29-III1968, Wilbur E Stone 10289 (DUKE, F, MO). Limón: Cordillera de Talamanca, cabeceras del río Teribe, III-1984, Davidse \& al. 25763 (CR, MO). Puntarenas: Zona Protectora Las Tablas, Las Alturas, 19-VII-1999, Alfaro 2397 (INB, MO); sendero a Cerro Echandi, 16-V-2000, Alfaro 3171 (INB); Monteverde, 9-XI-1985, Bello 3276 (CR, EAP, MEXU, MO); Monteverde, VI-1993, Bradford 99 (MO); entre Cerro Echandi y Cerro Burú, 24-VIII-1983, Davidse E al. 24012 (CR, MO); Parque Internacional La Amistad, puesto Tres Colinas, 15-IX-1996, Gamboa E Picado 582 (INB, MO); sendero a Cerro Echandi, 14-VIII-1997, Gamboa \& al. 1763 (CR, INB, MO); reserva biológica Monteverde, 29-VI-1993, Haber E Zuchowski 11517 (CR, INB, MO); Buenos Aires, Ujarrás, 6-X1989, Valerio 200 (CR, F). San José: Cerro Las Vueltas, 26-II-1965, Godfrey 66700 (MO); La Palma, XII.1913, Jiménez 949 (CR); Cedral, Alto Hierbabuena, 23-IV-1993, Jiménez E al. 1290 (INB, MO); quebrada Bajo Máquina, NE de Cascajal, 29-VIII-1971, Lent 2100 (EAP, F); SW del El Empalme, 19-XII-1963, Jiménez 1418 (F); Dota, 1947, Madriz 47 (F); Cerros de Escazú, Cedral, 9-X-1991, Morales 158 (CR, MO); Cuerecí, 3-VI-1996, Picado 433 (CR, INB, MO); Cerros de Escazú, Cedral y Alto Hierbabuena, 1-X-1993, 
Ramírez E Morales 135 (CR, INB, MO); Zona Protectora Cerros de Escazú, río Poás, 11-IX-1993, Morales 1687 (INB, MO); Acosta, Fila Bustamante, Tiquires, 3-VI-1995, Morales 4301 (INB); División, San Isidro del General, 6-II-1963, Williams E al. 24414 (CR, EAP, F); N de San Isidro del General, Cordillera de Talamanca, 29I-1965, Williams \& al. 28505 (EAP, F).

PANAMÁ. Chiriquí: Cerro Copete, 29-VII-1947, Allen 4892 (EAP, F, G, MO, S); Alto Bambito, 8 -IV-1979, D'Arcy \& al. 13107 (MO); Volcán Chiriquí, 19-XI-1975, Davidse \& D’Arcy 10197 (MO); Volcán de Chiriquí, 15-VII-1938, Davidson 977 (F); Volcán Barú, 30-XII-1985, de Nevers E Charnley 6682 (MO); Volcán Barú, 5-V-1978, Hammel 2820 (MO); volcán Barú, 24-VII-1975, Mori E Bolten 7418 (MO); Volcán Barú, 2-V-1983, Schmalzel E Schupp 1517 (MO); valle del río Chiriquí Viejo, cercanías de Monte Lirio, 27-VI/13-VII-1935, Seibert 288 (MO); Volcán Barú, W de Boquete, 8-I-1983, Stein 1285 (MEXU, MO); Bugaba, Santa Clara, Cerro Pando, 28-II-1985, van der Werff 7201 (MO); entre Nueva Suiza y Bambito, 27-V-1970, Wilbur \& al. 11880 (DUKE, MO); valle del río Chiriquí, 13-I-1971, Wilbur \& al. 13037 (DUKE, F); río Chiriquí Viejo, N de Cerro Punta, 25-XII-1971, Wilbur E al. 15328 (DUKE, F, MO). San Blas: Cerro Brewster, 21-IV-1985, de Nevers \& al. 5425 (MO).

9. Weinmannia vulcanicola J.F. Morales, Novon 15(2): 329, f. 2.2005

Tipo: Costa Rica. Alajuela: Grecia, Parque Nacional Volcán Poás, sendero a La Laguna Botos, 28-IX1999 (fl), J.F. Morales 7183 (holótipo, INB; isótipos, K, MO). Fig. 10.

Arbustos de 2-8 m de altura, los tallos densamente tomentosos o tomentulosos, indumento ferrugíneo a marrón ferrugíneo, algo glabrescentes con la edad. Hojas compuestas, con (11)15-25(29) foliolos; lámina (6) 8-15 × 3-8 mm, elíptica, subcoriácea, usualmente glabra, con sólo pelos a lo largo del nervio central abaxialmente, la mayoría con el ápice obtuso a redondeado (raramente agudo), la base obtusa a redondeada, los márgenes crenado-serrados, variadamente cóncavos y revolutos; entrenudos del raquis alados, con un ancho de 1,5-2,5(3) $\mathrm{mm}$ de ancho, el raquis densamente tomentoso o tomentuloso abaxialmente, indumento ferrugíneo a marrón ferrugíneo; peciolos 4-6 $\mathrm{mm}$, usualmente no alados, densamente tomentosos; estípulas 6-7 $\times 8-9 \mathrm{~mm}$, anchamente ovadas. Inflorescencias 6-12 cm de largo, el raquis y los pedicelos esparcidamente puberulentos, el indumento ferrugíneo o marrón-ferrugíneo, diminuto; flores $2,5-3 \mathrm{~mm}$ de largo, pedicelos 2-4 mm de largo, lóbulos del cáliz 0,7$1 \mathrm{~mm}$ de largo, glabros o glabrescentes, el ápice cortamente acuminado, pétalos ca. $2 \mathrm{~mm}$ de largo, ovario 1,5-2 mm de largo, glabro. Cápsulas de 4-5 mm de largo, glabras.

Distribución, hábitat y ecología. Endémica de Costa Rica, en las Cordilleras Central y de Talamanca, donde crece en robledales, bosques nubosos y bordes de sabanas, en elevaciones de 2300-3000 m. Especíme- nes con flores han sido recolectados entre febrero y octubre. Especímenes con frutos se recolectaron entre agosto y diciembre. Es probable que esta especie se encuentre en el W de Panamá, en la continuación de la Cordillera de Talamanca.

Weinmannia vulcanicola puede confundirse con W. intermedia Schltdl. \& Cham., ya que ambas comparten hojas con similar número de foliolos, pero la primera especie se puede separar con facilidad por sus foliolos usualmente cóncavos y con los márgenes revolutos, así como el indumento ferrugíneo o caféferrugíneo presente en las ramitas e inflorescencias. Weinmannia vulcanicola fue conocida anteriormente como W. trianaea Wedd., pero ambas especies difieren por diversos caracteres de las hojas y coloración del indumento de las partes vegetativas y florales (Morales, 2005).

\section{Especímenes examinados}

COSTA RICA. Alajuela: Volcán Poás, 11-IX-1965, Bernardi 10535 (F, G, MO, Z); Volcán Poás, 21-IV-1968, Córdoba 918 (CR, EAP); Poás, 1930, Cufodontis 220 (W); Parque Nacional Volcán

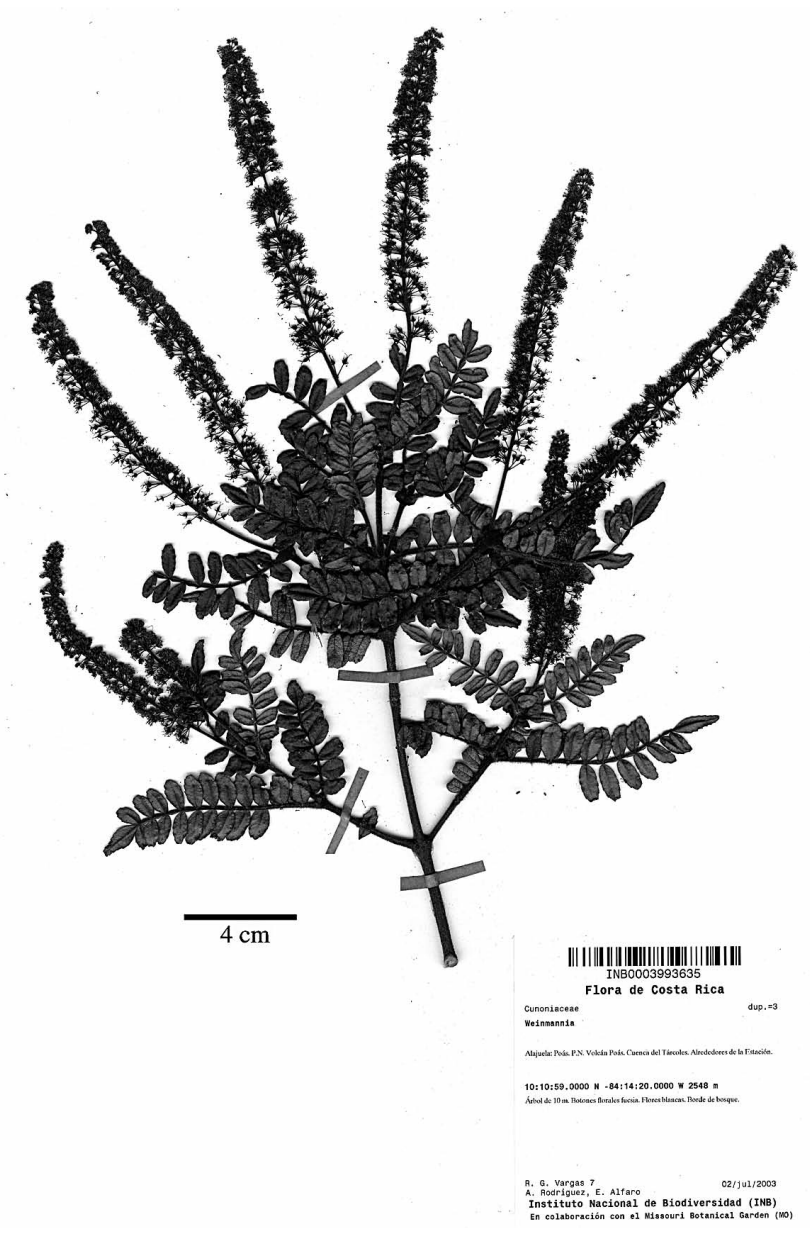

Fig. 10. Weinmannia vulcanicola (Vargas et al. 7, INB). 
Poás, 5-V-1991, Döbbeler E Dauphin 5488 (CR); Monteverde, 30XI-1976, Dryer 1046 (F, MO); Volcán Poás, 11-XI-1967, Espinoza 13 (CR); Parque Nacional Volcán Poás, 3-III-1999, Gargiullo 815 (CR); Fila Volcán Viejo, San Carlos, II-1986, Gómez-Laurito 11032 (CR, F, USJ); Volcán Poás, 2-IV-1950 (st), Gregory 6 (EAP); Volcán Poás, 18-V-1965, Hatheway 1339 (CR); volcán Poás, 16III-1964, Jangoux 1349 (BR); Volcán Poás, 19-VIII-1961, Jiménez 193 (CR, F), 11-XI-1967, Jiménez E Rodríguez 384 (CR), II-1915, Jiménez 1017 (CR, F, US); Parque Nacional Volcán Poás, 25-X1964, Little E Budowski 20020 (CR); Volcán Poás, 20-VIII-1965, McDaniel 6718 (MO); Volcán Poás, 11-XI-1967, Orozco 13 (CR); Laguna superior del Poás, 31-VIII-1890, Pittier 2972 (BR); Volcán Poás, 18-VI-1971, Proctor 32468 (F, MO); Parque Nacional Volcán Poas, 19-VII-1976, Solomon 5320 (MO); volcán Poás, IX.1896, Tonduz 10780 (BR, W); Volcán Poás, 13-VII-1962, Webster E al. 12280 (F, MO, S, U); W de Vara Blanca, 21-I-1968, Wilbur \& Stone 9837 (DUKE, F, MO); Volcán Poás, 1-IX-1968, Wilbur \& Stone 10650 (DUKE, F, MO). Heredia: Vara Blanca, 31XII-1973, Almeda \& al. 2206 (DUKE, F, MEXU); Parque Nacional Braulio Carrillo, Volcán Barva, 13-VIII-1993, Boyle E al. 2360 (CR, MO), 14-VIII-1993, Boyle E al. 2382 (MO); entre Sacramento y Laguna de Barva, Volcán Barva, 12-IV-1975, Utley \& Utley 2027 (F). Limón: Talamanca, Sabanas de Dúrika, río Kuk, 20-X1989, Chacón 584 (CR, F, INB, MO); Cordillera de Talamanca, Cerros Tararia, 10-IX-1984, Davidse E al. 28848 (BM, INB, MEXU, MO); Parque Internacional La Amistad, Cerros Tararia, Valle del Silencio, 1-IV-1997, Quesada E al. 1924 (INB).

10. Weinmannia wercklei Standl., J. Wash. Acad. Sci. 17(12): 309. 1927

Tipo: Costa Rica. Cartago: San Cristóbal de Candelaria, 1700 m, s.d. (fr), Werckle s.n. [Pittier 3685] (holótipo, US; isótipo, BR). Fig. 11.

Weinmannia tonduzii Engl., Nat. Pfl. Syst. (ed. 2) 18a: 252. 1930

Tipo: Costa Rica. San José: La Palma, 1450 m, fecha perdida (fl), Biolley E Tonduz 12513 (holótipo, B; isótipos, $G, M, U S)$.

Árboles o arbustos 4-20 m de altura, los tallos tiernos moderada a esparcidamente puberulentos, el indumento del mismo color de las ramitas, con la edad glabros o glabrescentes. Hojas simples; lámina 25-50 $\times 10-20(25) \mathrm{mm}$, angostamente elíptica, membranácea, glabra o glabrescente, el ápice agudo o acuminado, la base cuneada o decurrente, los márgenes crenado-serrados, raramente serrados, no revolutos; peciolos hasta $3 \mathrm{~mm}$ de largo, no alados, glabrescentes o glabros; estípulas 4-7 × 4-5 mm, anchamente ovadas. Inflorescencias $3-6,5 \mathrm{~cm}$ de largo, el raquis y los pedicelos esparcidamente puberulentos, el indumento no ferrugíneo ni amarillento, diminuto; flores $2-2,5 \mathrm{~mm}$ de largo, pedicelos 2-4,1 mm de largo, lóbulos del cáliz ca. $0,8 \mathrm{~mm}$ de largo, esparcidamente puberulentos, el ápice agudo, pétalos 1,6-2 mm de largo, ovario ca. 1 $\mathrm{mm}$ de largo, tomentuloso. Cápsulas 2-2,5(3) mm de largo, diminuta y esparcidamente puberulentas, algunas veces glabrescentes.
Distribución, bábitat y ecología. Endémica de Costa Rica y el W de Panamá, donde crece en bosques muy húmedos, bosques nubosos y ventosos, así como en vegetación secundaria relacionada, entre los 700-1700 m. La floración ocurre de mayo a noviembre. La fructificación se ha reportado de noviembre a marzo.

Weinmannia wercklei puede ser confundida con W. balbisiana Kunth, pero esta última especie tiene hojas usualmente más anchas, así como frutos ligeramente más largos. Las hojas de especímenes estériles algunas veces tienen láminas foliares mucho más grandes que las aquí mencionadas, pero tal y como se mencionó en la introducción, especímenes estériles pueden ser irregulares en relación al típico patrón de un determinado carácter encontrado en una especie. Por lo general el borde es crenado-serrado, sin embargo, ocasionalmente se encuentran especímenes atípicos con hojas conspicuamente serradas.

\section{Especímenes examinados}

COSTA RICA. Alajuela: Reserva Biológica Monteverde, 3-I1990, Bello 1667 (INB, MO); río Peñas Blancas, quebrada El Valle, 17-VIII-1993, Bello E Cruz 5225 (CR, INB, MO); La Palma de San

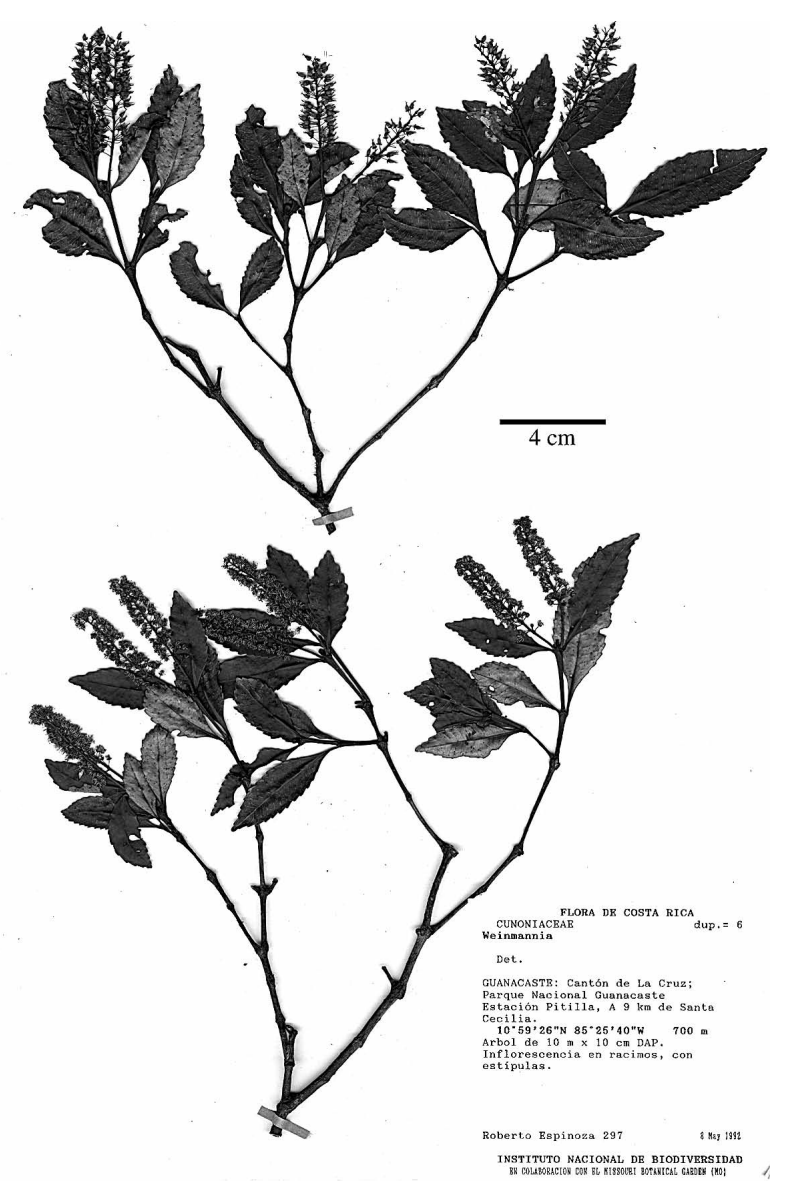

Fig. 11. Weinmannia wercklei (Espinoza 297, INB). 
Ramón, 2-XI-1924, Brenes 4132 (CR, F), 2-VIII-1925, Brenes 4372 (CR, F), 8-XI-1925, Brenes 4602 (CR, F, G), 5-X-1927, Brenes 5721 (CR, EAP, F); río La Paz, 8 -IV-1974, Hartshorn 1437 (CR, F, MO); San Ramón, NW de Cedral de Montes de Oro, 13-XI-1993, Jiménez E al. 1414 (CR, INB, MO); Monteverde, 23-VIII-1987, J.F. Smith 546 (F), Tapezco, Zarcero, 12-IX-1938, A. Smith 1180 (F, MO, NY). Cartago: Orosi, Mirador Monte Sky, 24-IX-1995, Aguilar E al. 4405 (CR, INB, MO); zona protectora Río Navarro y Río Sombrero, 4-XII-2000, Alfaro 3525 (INB); El Guarco, 9-XII-1966, Gónzalez 33 (F); Parque Nacional Tapantí, Alto Jaular, 19-VII1994, Morales E al. 3035 (CR, INB, MO); El Muñeco, 1926, Standley E Torres 51140 (US). Guanacaste: Parque Nacional Guanacaste, Volcán Cacao, 3-XII-1989, Chávez E Blanco 18 (MO); Parque Nacional Guanacaste, estación Pitilla, 8-V-1992, Espinoza 297 (CR, INB, MO); La Cruz de Abangares, 4-IX-1985, Haber \& Wolfe 2517 (CR, EAP, MO); Monteverde, camino a Las Nubes, 10-XI-1988, Haber E Zuchowski 8733 (CR, MEXU, MO); Parque Nacional Guanacaste, Cerro Orosilito, 14-VI-1989, Jiménez \& al. 702 (CR, MEXU, MO); Parque Nacional Guanacaste, estación Pitilla, 20-V1992, Moraga 454 (INB). Heredia: Parque Nacional Braulio Carrillo, sendero del transecto, 15-VIII-1992, Boyle 998 (CR, MO); Barva, La Legua, 9-X-1992, Boyle \& al. 1126 (MO); río Para Blanca, Cerro de Zurquí, 21-X-1975, Burger E al. 9354 (CR, F, MO); cerros de Zurquí, 1926, Standley \& Valerio 50589 (US). Limón: Bratsi, Alto Lari, 15-III-1992, Aguilar E Schmidt 1114 (CR, INB, MO). Puntarenas: Arancibia de Miramar, 4-X-1989, Bello 1336 (CR, INB, MO); Monteverde, 15-XI-1985, Bello 3334 (CR, EAP, MO); Cerro Plano, Monteverde, 1-VIII-1993, Bradford 96 (MO); Monteverde, 27-VII-1983 (fl), Costich E Baldwin 1535 (F); Monteverde, 9-III-1976, Dryer 745 (CR, MO), 30-XI-1976, Dryer 1046 (CR, F, MO); Monteverde, 21-VIII-1984, Gentry \& Haber 48744 (MO); Monteverde, 29-VIII-1985, Haber 2438 (C, MO); Monteverde, 11X-1985, Haber 3068 (CR, EAP, MO); Monteverde, 13-X-1986, Haber \& Bello 5934 (C, CR, MO); Cerro Ojo de Agua, Monteverde, 23-I-1987, Haber \& Bello 6615 (CR, F, MO); cuenca de los ríos Lagarto y Guacimal, reserva Monteverde, 18-X-1995, Haber 11977 (INB, MO); Monteverde, SE de Cerro Amigos, 19-VIII-1995, Penneys E Zuchowski 682 (CR, INB, MO). San José: Bajo La Hondura, 20-VI-1973, Poveda 571 (CR); Santa María de Dota, 1926 Standley 41581 (US). Localidad perdida: -IV-1910, Worthen s.n. (MO)

PANAMÁ. Bocas del Toro-Chiriquí: Cerro Colorado, 16VIII-1977, Folsom 4884 (MO).

\section{Agradecimientos}

Los herbarios BIGU, BM, BR, C, COL, CR, DUKE, EAP, ENCB, F, G, INB, M, MA, MEXU, MHES, MO, NY, P, QCNE, S, TEFH, U, US, W, WIS, WU, Z facilitaron el estudio de sus colecciones. Quiero agradecer la colaboración de Gerrit Davidse y el Missouri Botanical Garden (MO) a través del Proyecto Flora Mesoamericana al facilitar el estudio de especímenes de Cunoniaceae en el año 2004, que permitió completar este trabajo y el tratamiento de esta familia para dicho proyecto. Barry Hammel (MO) volvió a prestar su colaboración en la revisión del resumen en inglés y Michael Grayum (MO) suministró referencias bibliográficas claves y resolvió dudas de diversa índole. Finalmente, quedo agradecido a las siguientes personas por facilitar la visita de sus herbarios: Julio Betancur (COL), Philip Silverstone-Sopkin (CUVC), Rocío Rojas (HOXA), Ricardo Callejas, Ramiro Fonnegra y Javier Francisco Roldán (HUA), Álvaro Cogollo (JAUM), Jorge Monterrosa (LAGU), Eunice Echeverría (MNES), Homero Vargas (QCNE), Cirilo Nelson (TEFH), Joaquina Alban y Asunción Cano (USM), Shingo Nozawa, Sirly Leithon y Eliana Nogueira (VEN), Mary y Peter Endress (Z).

\section{Referencias bibliográficas}

Bernardi, L. 1961. Revisio generis Weinmanniae. Pars I: secio Weinmanniae. Candollea 17: 123-189.

Bernardi, L. 1961. Revisio generis Weinmanniae. Pars I: secio Weinmanniae. Candollea 17: 123-189.

Bradford, J. 1998. A cladistic analysis of species groups in Weinmannia (Cunoniaceae) based on morphology and inflorescence structure. Ann. Missouri Bot. Gard. 85: 565-593.

Bradford, J. 2002. Molecular phylogenetics and morphological evolution in Cunonieae (Cunoniaceae). Ann. Missouri Bot. Gard. 89: 491-503.

Bradford, J. \& P.E. Berry. 1998. Cunoniaceae. In: Steyermark, J.A., Berry, P.E. \& Holst, B. (eds.), Fl. Venez. Guayana 4: 462469.

Britton, N.L. 1905. Cunoniaceae, Iteaceae. In: Britton, N. \& Underwood, L.M. (eds.), North American flora 22(2): 179-181. New York Bot. Gard., New York, NY, EUA.

Cuatrecasas, J. 1948. Studies in South American Plants I. Lloydia 11: $185-225$

Harling, W. 1999. Cunoniaceae. In: Harling, G. \& Anderson, L. (eds.), Fl. Ecuador 61: 1-74.

Morales, J.F. 2005. Nuevas especias de Weinmannia (Cunoniaceae) para Costa Rica y Colombia. Novon 15(2): 327-331.

Stein, B.A. 2001. Cunoniaceae. In: Stevens, W., Ulloa Ulloa, C., Pool, A. \& Montiel, O.M. (eds.), Flora de Nicaragua. Monogr. Syst. Bot. Missouri Bot. Gard. 85: 718.

Woodson, R. \& R. Schery. 1950. Cunoniaceae. In: Woodson Jr., R.E., Schery, R. W. \& al. (eds.), Flora of Panama, Part V, Fascicle 2. Ann. Missouri Bot. Gard. 37: 145-147.

\section{Apéndice 1}

\section{Índice de nombres científicos}

Weinmannia anisophylla Standl. \& L.O Williams

Weinmannia balbisiana Kunth

Weinmannia balbisiana var. laurina (Kunth) Cuatrec.

Weinmannia burserifolia Standl.

Weinmannia fagaroides Kunth

Weinmannia glabra L.f.

Weinmannia horrida J.F. Morales

Weinmannia intermedia Schltdl. \& Cham.

Weinmannia intermedia Schltdl. \& Cham. var. pittieri Pamp.

Weinmannia karsteniana Szyszyl.

Weinmannia laurina Kunth

Weinmannia laurina var. pseudolaurina (Woodson) Bernardi

Weinmannia liebmannii Engl.

Weinmannia myrtifolia Cuatrec.

Weinmannia pinnata $\mathrm{L}$.

Weinmannia pseudolaurina Woodson

Weinmannia tonduzii Engl.

Weinmannia tuerckheimii Engl.

Weinmannia vulcanicola J.F. Morales

Weinmannia wercklei Standl.

\section{Apéndice 2}

\section{índice de ejemplares examinados}

Weinmannia anisophylla Standl. \& L.O Williams

Weinmannia balbisiana Kunth

Weinmannia burserifolia Standl.

Weinmannia fagaroides Kunth 
Weinmannia horrida J.F. Morales

Weinmannia intermedia Schltdl. \& Cham.

Weinmannia karsteniana Szyszyl.

Weinmannia pinnata L.

Weinmannia vulcanicola J.F. Morales

Weinmannia wercklei Standl.

\section{Apéndice 3}

\section{Índice de pliegos de herbario}

Aguilar, R., 1181 (4).

Aguilar, R. \& O. Garrote, 3996 (4).

Aguilar, R. \& H. Schmidt, 1114 (10).

Aguilar, R. \& al., 3933 (4); 4405 (10).

Alfaro, E., 92 (4); 2397 (8); 3171 (8); 3278 (2); 3279 (3); 3525 (10); $3700(2) ; 4654$ (5).

Alfaro, E. \& M. Alfaro, 2253 (7).

Allen, P., 2656 (2); 4736 (3); 4892 (8).

Allen, P. \& R. Armour, 7286 (2).

Almeda, F. \& al., 3347 (3).

Almeda, F. \& T. Daniel, 7211 (7).

Almeda, F. \& K. Nakai, 4808 (4).

Almeda, F. \& al., 2206 (9); 3347 (3).

Anderson, W. \& S. Mori, 67 (4).

Aranda, J. \& al., 3100 (5).

Beaman, J., 6226 (6).

Bello, E., 1336 (10); 1338 (3); 1560 (3); 1667 (10); 3276 (8); 3334 (10).

Bello, E. \& E. Cruz, 5225 (10).

Bernardi, L., 10535 (9).

Biolley, P. \& A. Tonduz, 12513 (10).

Boyle, B., 745 (4); 756 (8); 854 (3); 920 (3); 998 (10).

Boyle, B. \& A. Boyle, 486 (6); 511 (6); 639 (6).

Boyle, B. \& P. Hibbs, 960 (3); 3175 (3).

Boyle, B. \& al., 1126 (10); 2358 (4); 2359 (4); 2360 (9); 2363 (4); 2367 (4); 2368 (4); 2382 (9); 2384 (4); 5437 (4).

Bradford, J., 96 (10); 97 (3); 99 (8).

Bradford, J. \& al., 2363 (4).

Breedlove, D., 12440 (8); 19958 (3); 31418 (3).

Breedlove, D. \& P. Raven, 1288612440 (8).

Breedlove, D. \& A. R. Smith, 22029 (3).

Brenes, A., 4132 (10); 4372 (10); 4602 (10); 5665 (8); 5721 (10).

Burger, W. \& R. Baker, 9496 (8).

Burger, W. \& al., 9354 (10); 12399 (2).

Calzada, J., 5240 (6); 5425 (6).

Carlson, M., 3619 (4).

Castillo, J. \& A. Vásquez, 1536 (6).

Cedillo, R. \& D. Lorence, 672 (6); 1266 (6).

Cedillo, R. \& R. Torres, 1867 (2).

Cedillo, R. \& al., 1644 (6)

Chacón, A., 328 (5); 584 (9).

Chávez, J. \& R. Blanco 18 (10).

Cházaro, M., 1188 (6).

Cházaro, M. \& H. Narave, 1680 (6).

Cházaro, M. \& L. Robles, 3109 (6); 3644 (6).

Churchill, H., 5518 (2).

Córdoba, J., 918 (9).

Correa, M. \& al., 4662 (3).

Costich, D. \& D. Baldwin, 1535 (10).

Croat, T., 325 (4); 26930 (3); 35008 (3).

Cruz, M., 137 (7); 184 (3)

Cuatrecasas, J., 9451 (7).

Cufodontis, G., 220 (9).

D'Arcy, W., 6446 (3); 11058 (3); 17892 (2);17955 (4).

D’Arcy, W. \& B. Hammel, 12501 (4).
D’Arcy, W. \& al., 13107 (8); 13215 (3).

Darío, D., 127 (1); 128 (2); 144 (1).

Davidse, G., 24864 (4); 34273 (2).

Davidse, G. \& W.D’Arcy, 10197 (8).

Davidse, G. \& G. Herrera, 29261 (3); 29309 (4).

Davidse, G. \& R. Zúñiga, 34823 (4).

Davidse, G. \& al., 24012 (8); 25128 (2); 25193 (2); 25763 (8); 25856 (4); 28848 (9); 28956 (2)

Davidson, C., 977 (8); 7246 (4).

de Nevers, G. \& S. Charnley, 6682 (8).

de Nevers, G. \& al., 5425 (8).

Döbbeler, P \& G. Dauphin, 5488 (9).

Dorantes, J., 588 (6).

Dryer, V. J., 745 (10); 1046 (10); 1565 (8).

Duke, J., 6568 (2).

Espinoza, R., 13 (9); 297 (10).

Estrada, A., 464 (7).

Fay, J. \& J. Calzada, 876 (6).

Fernández, A., 152 (3); 706 (3); 1459 (8); 1541 (4).

Folsom, J., 4884 (10).

Galeotti, H., 2837 (8); 2838 (6).

Gamboa, B., 1537 (4).

Gamboa, B. \& A. Picado, 582 (8).

Gamboa, B. \& al., 1249 (4); 1763 (8).

García, A. \& al., 1301 (6); 1843 (6); 5043 (2).

Gargiullo, M., 815 (9).

Gentry, A. \& W. Haber, 48744, (10).

Gentry, A. \& S. Mori, 13623 (2); 13987 (2).

Gentry, A. \& al., 16908 (2).

Gimate, J., 1077 (6).

Glassman, S., 1671 (2)

Godfrey, R., 66700 (8)

Gómez, L. D. \& al., 21876 (4).

Gómez-Laurito, J., 5274 (2); 6362 (7); 11032 (9).

González, J., s.n. (2), 33 (10); 632 (3); 655 (4).

Grayum, M. \& J. Affolter, 8168 (8).

Greenman, J. \& M. Greenman, 5607 (4).

Gregory, L., 6 (9).

Haber, W., 2438 (10); 3068 (10); 11977 (10).

Haber, W \& E. Bello, 5934 (10); 6615 (10).

Haber, W. \& M. Ramírez, 11680 (3).

Haber, W \& J. Wolfe, 2517 (10).

Haber, W \& W. Zuchowski, 8733 (10); 11517 (8).

Hagen, C. von \& W. von Hagen, 2068 (3).

Hamilton, C. \& K. Krager, 3879 (3).

Hamilton, C. \& H. Stockwell, 3362 (3).

Hammel, B., 2820 (8); 6082 (2); 7379 (2); 7554(2).

Hammel, B. \& J. González, 20884 (4).

Hammel, B. \& al., 6753 (3); 17650 (3).

Hartman, R., 12468 (2).

Hartshorn, G., 1150 (8); 1437 (10).

Hatheway, W., 1339 (9).

Hawkins, T., 72 (4).

Hawkins, T. \& al., 353 (4); 592 (2); 733 (2).

Hazlett, D., 1900 (2); 2231 (4); 2506 (2).

Hazlett, D. \& F. Coe, 2879 (4).

Heed, W., s.n. (2).

Hernández, H., 1463 (6); 1473 (6); 1557 (6); 3435 (6).

Hernández, M. \& Sharp, 96, (6).

Herrera, G. \& al., 888 (2).

Hill, S. R. \& al., 17889 (4).

Holm, R. \& H. Iltis, 148 (4).

House, P., 1034 (2).

Humboldt, A. \& A. Bonpland, s.n. (2); s.n. (2); s.n. (4).

Ishiki, M., 1296 (8).

Jangoux, J., 1349 (9). 
Jiménez, A., 193 (9); 1017 (9).

Jiménez, A \& R. Rodríguez, 384 (9).

Jiménez, Q., 747 (3); 949 (8); 1418 (8).

Jiménez, Q. \& al., 556 (2); 702 (10); 1290 (8); 1414 (10); 1484 (4).

Kappelle, M., 320 (7).

Kriebel, R., 958 (4).

Kupper, W., 1310 (4).

Lawton, R., 792 (6).

Lent, R., 1197 (4); 1461 (2); 2100 (8).

León, J., 283 (8).

Lépiz, E. \& J.F. Morales, 272 (4).

Lépiz, E. \& V.H. Ramírez, 393 (7).

Liebmann, C., 1776 (3); 1777 (3); 1778 (3); 1779 (3).

Little, E. \& G. Budowski, 20020 (9).

López, R. \& G. Martin, 371 (6).

Lundell, C. \& E. Contreras, 20907 (2); 20930 (2); 21974 (8).

Madriz, F., 11 (7); 47 (8); 59 (7).

Márquez, W., 486 (6).

Márquez, W. \& J. Dorantes, 114 (6).

Martin, G., 499 (6).

Martínez, R., s.n. (2).

Matuda, E., 4285 (8); 5371 (8); 17851 (8).

McDaniel, S., 6718 (9).

McPherson, G., 7899 (2); 8403 (2); 15916 (3).

McPherson, G. \& M. Merello, 8332 (2); 8363 (3).

Mejía, D., 100 (4); 310 (4); 340 (2).

Molina, A., 1007 (2); 1018 (8); 10107 (2); 10669 (2); 12735 (8); 12740 (2); 13743 (1); 14500 (2).

Molina, A. \& A. Molina, 12665 (2).

Molina, A. \& al., 17848 (8).

Mora, E., 6 (3).

Moraga, M., 384 (5); 454 (10).

Morales, J.F., 158 (8); 1687 (8); 2589 (8); 3035 (10); 3112 (7); 3626 (2); 4301 (8); 7183 (9).

Morales, J.F. \& R. Abarca, 3099 (4); 3105 (4).

Morales, J.F. \& E. Lépiz, 2606 (4); 2636 (2); 3094 (4).

Morales, J.F. \& J. Quesada, 5817 (3).

Morales, J.F. \& al., 1609 (4); 2244 (3); 2673 (8); 5761 (2); 6980 (2).

Mori, S. \& A. Bolten, 7418 (8); 7686 (2).

Mori, S. \& R. Dressler, 7836 (2).

Mori, S. \& J. Kallunki, 5692 (3); 5820 (2).

Murphy, H., 1023 (3).

Nee, M. \& B. Hansen, 18662 (6).

Nee, M. \& G. Martin, 32261 (6).

Nee, M. \& W. Robleto, 28099 (8).

Nee, M. \& K. Taylor, 26901 (6).

Neill, D., 1844 (3); 3861 (3).

Neill, D. \& P. Vincell, 3305 (8).

Nelson, C. \& al., 19138 (1).

Omme, L. van, 234 (1).

Orozco, C., 13 (9).

Padgett, G. \& al., 266 (4).

Penneys, D \& W. Zuchowski, 682 (10).

Picado, A., 433 (8).

Pittier, H., 2972 (9); 3685 (10); 13045 (8).

Poveda, L., 571 (10); 625 (7).

Pringle, C., 8977 (6); 10026 (6).

Proctor, G., 31843 (3); 32468 (9).

Purpus, C., 5931 (6); 6975 (8).

Quesada, F. \& V. Sancho, 1819 (4).

Quesada, F. \& al., 1924 (9); 1992 (4).

Ramírez, E. \& P. Ramírez, 425 (8).

Ramírez, V.H., 454 (4).

Ramírez, V.H. \& J.F. Morales, 135 (8).

Reark, J.B., 207 (2).

Reyna, M., s.n. (2).
Rodríguez, A. \& P. Hurtado, 4410 (2).

Rivera, G., 155 (8); 163 (8).

Rivera, J. \& G. Martin, 1159 (8).

Robleto, W., 497 (8).

Schiede, C. \& F. Deppe, 521 (6).

Schmalzel, R., 1439 (3).

Schmalzel, R. \& J. Schupp, 1517 (8).

Seibert, R., 288 (8).

Sessé y Lacasta, M. \& J. Moçiño, 991 (6).

Skutch, A., 3299 (8).

Smith, A., 155 (3); 259 (8); 1180 (10); 2622 (1); 4219 (8).

Smith, J.F., 546 (10).

Solomon, J., 5320 (9).

Stafford, P. \& al., 339 (8).

Standley, P., 41581 (10); 68536 (8); 86317 (8); 86446 (8); 92313 (2).

Standley, P. \& R. Torres, 51140 (10).

Standley, P. \& J. Valerio, 49848 (3); 50589 (10).

Stein, B., 1285 (8).

Stern, W. \& al., 33786 (3).

Stevens, W. D., 6498 (8); 11327 (3); 14056 (8).

Steyermark, J., 42617 (2); 43276 (1); 43297 (8); 43686 (2); 49002 (2).

Taylor, R.J. \& C. Taylor, 4344 (4).

Taylor, C. \& M. Nee, 265 (6).

Thomas, D., 733 (2).

Tonduz, A., 1950 (4); 10780(9).

Torres, R. \& L. Cortés, 8686 (6).

Torres, R. \& C. Martínez, 12532 (8).

Tucker, J., 1201 (2).

Tuerckheim von, H., 906 (2).

Tyson, E., 5843 (3).

Utley, J. \& K. Utley, 2027 (9); 3596 (7).

Valerio, J., 200 (8); 1263 (3); 1364 (8).

Varela, G., 233 (8).

Vargas, G. \& al., 1390 (2); 1415 (4).

Vargas, Y., 209 (6); 349 (6).

Vásquez, A. \& al., 4641 (6).

Ventura, F., 1089 (6); 3676 (6); 8582 (6); 8968 (6); 12885 (6); 20318 (6).

Webster, G., 22129 (4).

Webster, G. \& al., 12280 (9); 17462 (6).

Werckle, C., s.n. (10).

Werff, H. van der, 7201 (8).

Werff, H. van der \& H. Herrera, 6445 (2).

Wiemann, M., 279 (4); 280 (4); 293 (3).

Wilbur, R., 26067 (7).

Wilbur, R. \& J. Luteyn, 18804 (8).

Wilbur, R. \& D. Stone, 8770 (4); 9837 (9); 10056 (8); 10289 (8); 10650 (9)

Wilbur, R. \& J. Teeri, 13911 (7).

Wilbur, R. \& al., 11880 (8); 11963 (3); 13037 (8); 15328 (8); 22882 (3).

Williams, L., 17526 (1).

Williams, L. \& A. Molina, 12739 (2); 12800 (8); 13736 (2); 13917 (4).

Williams, L. \& R. Williams, 18910 (1).

Williams, L. \& al., 23064 (8); 24414 (8); 24456 (4); 25658 (8); $28210(8) ; 28505$ (8).

Wilson, M., 40790 (8).

Woodson, R. \& al., 821 (3).

Worthen, G., s.n. (10).

Zamora, N., 1119 (2).

Editor asociado: C. Ulloa Recibido: 23-II-2010 Aceptado: 13-X-2010 